ARTICLE

DOI: $10.1038 /$ s41467-018-05768-3

\title{
The tyrosine phosphatase SHP2 controls TGF $\beta$ - induced STAT3 signaling to regulate fibroblast activation and fibrosis
}

Ariella Zehender', Jingang Huang ${ }^{1}$, Andrea-Hermina Györfi', Alexandru-Emil Matei (1) 1, Thuong Trinh-Minh', Xiaohan Xu', Yi-Nan Li ${ }^{1}$, Chih-Wei Chen ${ }^{1}$, Jianping Lin ${ }^{2}$, Clara Dees ${ }^{1}$, Christian Beyer ${ }^{1}$, Kolja Gelse $^{3}$, Zhong-Yin Zhang ${ }^{2}$, Christina Bergmann ${ }^{1}$, Andreas Ramming ${ }^{1}$, Walter Birchmeier ${ }^{4}$, Oliver Distler ${ }^{5}$, Georg Schett ${ }^{1} \&$ Jörg H.W. Distler ${ }^{1}$

Uncontrolled activation of TGF $\beta$ signaling is a common denominator of fibrotic tissue remodeling. Here we characterize the tyrosine phosphatase SHP2 as a molecular checkpoint for TGF $\beta$-induced JAK2/STAT3 signaling and as a potential target for the treatment of fibrosis. TGF $\beta$ stimulates the phosphatase activity of SHP2, although this effect is in part counterbalanced by inhibitory effects on SHP2 expression. Stimulation with TGF $\beta$ promotes recruitment of SHP2 to JAK2 in fibroblasts with subsequent dephosphorylation of JAK2 at Y570 and activation of STAT3. The effects of SHP2 on STAT3 activation translate into major regulatory effects of SHP2 on fibroblast activation and tissue fibrosis. Genetic or pharmacologic inactivation of SHP2 promotes accumulation of JAK2 phosphorylated at Y570, reduces JAK2/STAT3 signaling, inhibits TGF $\beta$-induced fibroblast activation and ameliorates dermal and pulmonary fibrosis. Given the availability of potent SHP2 inhibitors, SHP2 might thus be a potential target for the treatment of fibrosis.

\footnotetext{
${ }^{1}$ Department of Internal Medicine 3-Rheumatology and Immunology, Friedrich-Alexander-University Erlangen-Nürnberg (FAU) and University Hospital Erlangen, Ulmenweg 18, 91054 Erlangen, Germany. ${ }^{2}$ Department of Medicinal Chemistry and Molecular Pharmacology, Purdue University, 575 Stadium Mall Drive Indiana, West Lafayette 47907, USA. ${ }^{3}$ Department of Trauma Surgery, Friedrich-Alexander-University Erlangen-Nürnberg (FAU), Krankenhausstraße 12, 91054 Erlangen, Germany. ${ }^{4}$ Max Delbrück Center for Molecular Medicine (MDC), Robert-Rössle-Str. 10, 13092 Berlin, Germany. ${ }^{5}$ Department of Rheumatology, University Hospital Zurich, Gloriastrasse 25, 8091 Zurich, Switzerland. These authors contributed equally: Ariella Zehender, Jingang Huang. Correspondence and requests for materials should be addressed to J.H. (email: Jingang.Huang@uk-erlangen.de) or to J.H.W.D. (email: joerg.distler@uk-erlangen.de)
} 
F ibrotic diseases are characterized by an excessive accumulation of extracellular matrix, which destroys the physiological architecture of affected tissues and often leads to severe dysfunction of the involved organs. Fibrotic tissue responses can affect virtually every organ and can manifest either as local or systemic fibrotic disease. Systemic sclerosis (SSc) is a prototypical systemic fibrotic disease that can affect multiple organ systems including the skin, the lungs, the heart and the intestine ${ }^{1}$. Although most individual fibrotic diseases have a low incidence, fibrotic tissue responses in chronic disease are highly prevalent, constituting a major burden on modern societies accounting for up to $45 \%$ of deaths in the developed world 2,3 .

Fibroblasts are key effector cells in fibrotic diseases. Upon activation, resting fibroblasts can acquire a myofibroblast phenotype, which is characterized by expression of contractile proteins and enhanced release of extracellular matrix ${ }^{4}$. While myofibroblasts are only temporarily observed during physiological tissue remodeling, they remain stably activated in fibrotic diseases. Transforming growth factor- $\beta$ (TGF $\beta$ ) is a core pathway of fibroblast activation in physiologic and pathologic conditions and plays a central role for the persistent activation of fibroblasts in fibrotic diseases ${ }^{5-7}$. TGF $\beta$ signaling occurs only temporarily in wound healing, but remains active in fibrotic diseases. Fibroblasts isolated from patients with fibrotic diseases demonstrate a TGF $\beta$ biased gene expression signature. Moreover, prolonged activation of TGF $\beta$ signaling in mice by fibroblast-specific overexpression of constitutively active TGF $\beta$ receptor type I results in a systemic fibrotic disease, whereas targeted inhibition of TGF $\beta$ signaling ameliorates fibrosis ${ }^{1}$. Uncontrolled and prolonged activation of TGF $\beta$ signaling is thus sufficient and required to induce persistent fibroblast activation and tissue fibrosis ${ }^{8}$. Although the central role of TGF $\beta$ in the pathogenesis of fibrotic diseases is well established, it remains still enigmatic why TGF $\beta$ signaling is not appropriately terminated in fibrotic diseases. Identification of central checkpoints and re-establishment of effective feedback regulation of TGF $\beta$ signaling might offer potential targeted therapies for fibrotic diseases.

SHP2, encoded by the PTPN11 gene, is a ubiquitously expressed non-receptor tyrosine phosphatase (PTP). SHP2 contains two N-terminal Src homology 2 (SH2) domains, a catalytic PTP domain and a C-terminal tail with two tyrosyl phosphorylation sites ${ }^{9}$. While SHP2 is normally inactive in its basal state, binding to phosphotyrosyl residues of substrate proteins induces conformational changes that activate its phosphatase activity ${ }^{10}$. SHP2 plays a complex role in the regulation of multiple signaling cascades $^{11,12}$. SHP2 has been shown to modulate signaling pathways activated by growth factors such as platelet-derived growth factor (PDGF), epidermal growth factor (EGF), fibroblast growth factor (FGF) and insulin-like growth factor-1 (IGF-1), by interferons and by cytokines such as interleukin (IL)-3, IL-6, granulocyte-macrophage colony-stimulating factor (GM-CSF), as well as by peptide hormones such as erythropoietin (EPO) and insulin. SHP2 participates in signal transduction of various intracellular pathways including $\mathrm{RAS} / \mathrm{RAF} / \mathrm{mitogen}$-activated protein kinase (MAPK), Janus kinase/signal transducer and activator of transcription (JAK/STAT) and phosphatidylinositol-3 (PI3) kinase pathways ${ }^{11-13}$. However, SHP2 does not only modulate multiple pathways, but may act at multiple sites within a single signaling pathway to modulate the signal relay. For instance, SHP2 directly interacts with cytokine and growth factor receptors, but also binds to a variety of signaling intermediates such as GRB2, FRS2, JAK2, the p85 subunit of PI3 kinase, IRS1 and $G A B$ proteins to further modulate the signaling outcome ${ }^{14,15}$. This regulation at multiple levels enables SHP2 to generate a wide range of diverse effects in different cellular contexts. In most cases, SHP2-induced dephosphorylation diminishes the signaling intensity. However, SHP2 can also promote signaling, either by dephosphorylation of endogenous inhibitors at activating sites or by dephosphorylation of inhibitory tyrosine phosphorylation sites $^{16,17}$. Finally, SHP2 may not only modulate signaling by dephosphorylation of target proteins, but also in a phosphataseindependent manner ${ }^{18}$. Altered activity of SHP2 has been implicated in the pathogenesis of multiple diseases. Those include the Noonan syndrome and the Leopard syndrome with inherited mutations of the PTPN11 gene ${ }^{9,19}$. The activity of SHP2 is also altered in various types of tumors due to acquired mutations of PTPN11. In addition, changes in expression and activity of SHP2 have been implicated into the pathogenesis of autoimmune diseases such as systemic lupus erythematosus or rheumatoid arthritis $^{20-22}$.

In our study, we aimed to characterize the role of SHP2 in SSc. We characterize SHP2 as a molecular checkpoint of TGF $\beta$ signaling. SHP2 is required for the activation of JAK2 and STAT3 by TGF $\beta$. Inactivation of SHP2 prevents TGF $\beta$-induced JAK2/ STAT3 signaling, reduces fibroblast activation and ameliorates experimental fibrosis. These findings might have translational implications as potent inhibitors of SHP2 currently undergo clinical evaluation in cancer.

\section{Results}

TGF $\beta$ induces SHP2 activity. To investigate the role of SHP2 in the pathogenesis of SSc, we first analyzed the expression pattern of SHP2 in skin biopsies of SSc patients and healthy controls. The messenger RNA (mRNA) levels of SHP2 were modestly but statistically significantly decreased in fibrotic skin of SSc patients compared to matched healthy individuals (Fig. 1a). This downregulation was confirmed by immunohistochemistry (Fig. 1b) and immunofluorescence staining (Fig. 1c). Double staining with the fibroblast marker prolyl-4-hydroxylase- $\beta(\mathrm{P} 4 \mathrm{H} \beta)$, the endothelial marker CD31 and the leukocyte marker CD45 demonstrated that fibroblasts account for most of the SHP2 expression in the dermis and that SSc fibroblasts express reduced levels of SHP2 compared to fibroblasts in healthy skin (Fig. 1c). Quantification of the staining further confirmed the decrease of SHP2 in SSc fibroblasts compared to those in healthy skin (Fig. 1c). The mRNA (Fig. 1d) and protein levels (Fig. 1e) of SHP2 were also decreased in cultured SSc fibroblasts as compared to fibroblasts from healthy individuals. The expression of Shp2 was also modestly downregulated in murine models of SSc. The mRNA and protein levels of Shp 2 were decreased by $35-45 \%$ in the skin of bleomycinchallenged mice and in TSK1 mice. Co-staining of Shp 2 with vimentin demonstrated reduced expression of Shp2 in fibroblasts in fibrotic murine skin (Supplementary Fig. 1a-b).

We next investigated the molecular mechanisms underlying the decreased expression of SHP2 in fibrotic tissues. As activation of TGF $\beta$ signaling is a common denominator of fibrotic conditions, we analyzed potential effects of TGF $\beta$ on SHP2 expression. Indeed, stimulation of human fibroblasts with recombinant TGF $\beta$ reduced the $\mathrm{mRNA}$ and protein levels of SHP2 with maximal effects after $24 \mathrm{~h}$ and $72 \mathrm{~h}$, respectively (Fig. 2a, b). Furthermore, activation of TGF $\beta$ signaling by overexpression of a constitutively active TGF $\beta$ receptor type I (TBRICA) downregulated $\operatorname{Sh} p 2 \mathrm{mRNA}$ and protein levels in murine skin (Fig. 2c, d). In contrast, inhibition of TGF $\beta$ signaling by treatment with the selective TBRI inhibitor SD-208 prevented the downregulation of Shp2 in experimental fibrosis (Fig. 2e-h).

We next investigated the effects of TGF $\beta$ on SHP2 activity using phosphatase assays. In contrast to its inhibitory effects on SHP2 expression, TGF $\beta$ increased SHP2 activity in human dermal fibroblasts (Fig. 2i). Stimulation of SHP2 activity by TGF $\beta$ was observed as early as within $5 \mathrm{~min}$ and reached a plateau 
a

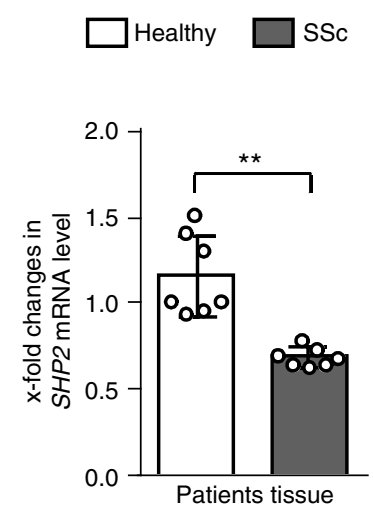

b

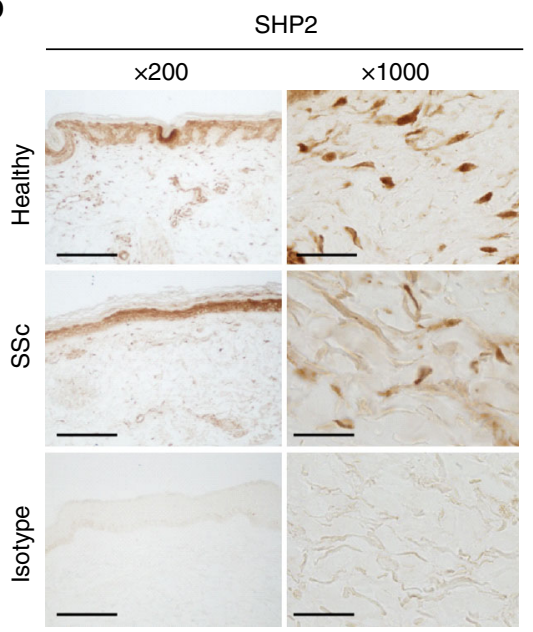

C
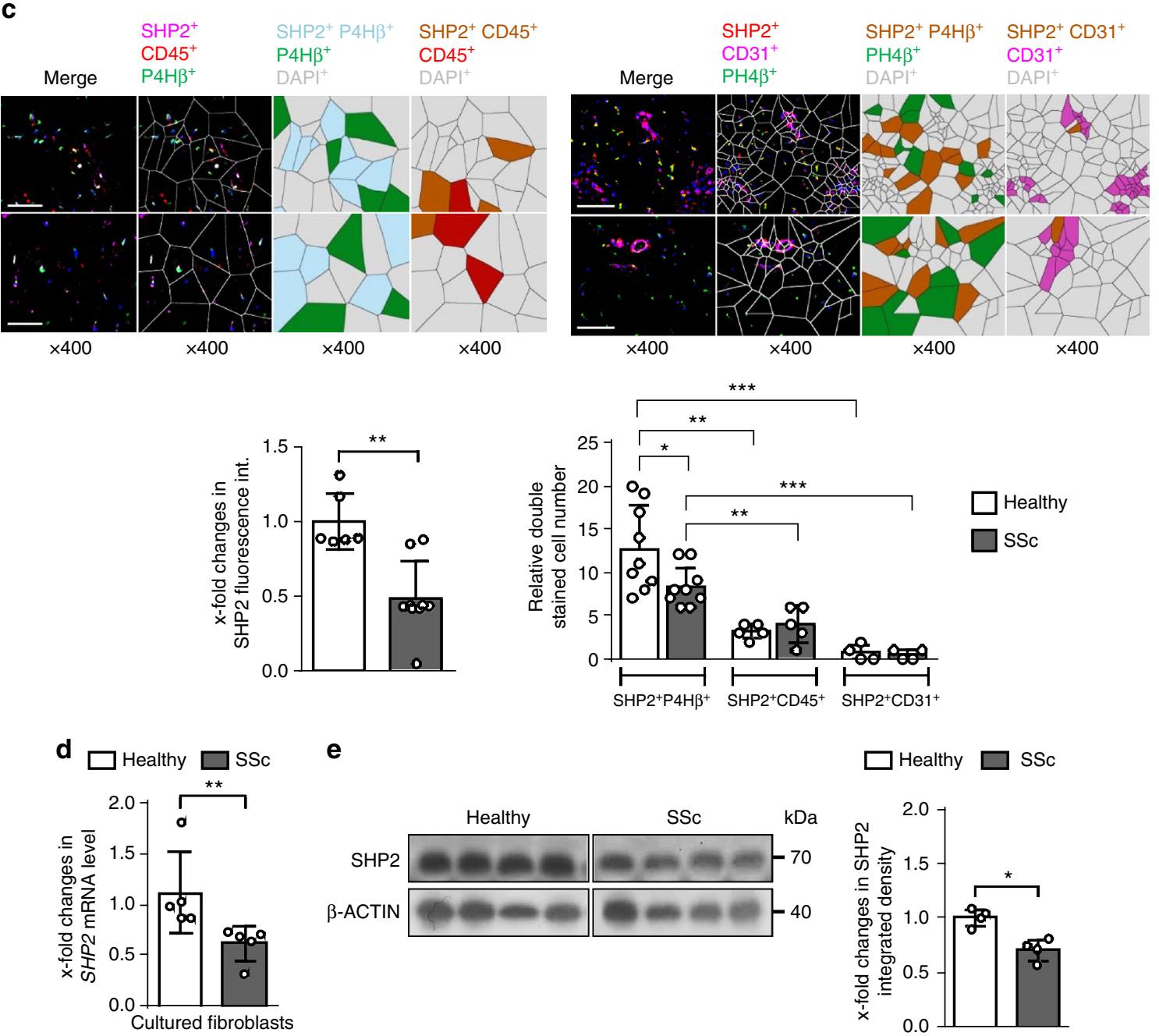

e

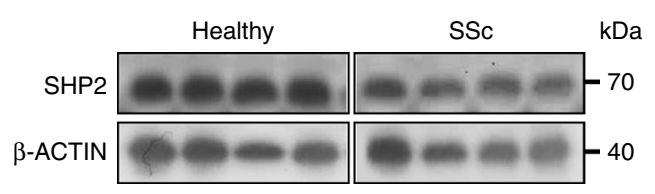

$\square$ Healthy

SSc

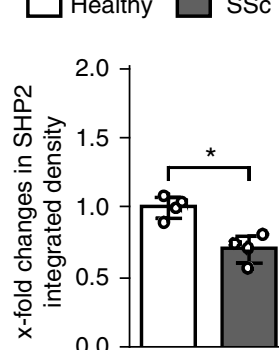

Fig. 1 Decreased expression of SHP2 in SSc. a The mRNA levels of SHP2 are significantly reduced in SSc skin as compared to healthy skin ( $n=7$ ). b Immunohistochemistry of SHP2 in SSc skin and matched healthy controls. Representative images are shown at 200- and 1000-fold magnification. c Immunofluorescence staining of SHP2 with co-staining for the fibroblast marker P4H $\beta$, the endothelial cell marker CD31 and the leukocyte marker CD45, and DAPI. SSc fibroblasts demonstrated a reduced staining for SHP2 compared to healthy control. Representative images are shown at 400-fold magnification. Immunofluorescence pictures were processed to generate Voronoi tessellated pictures amenable to computational simulation. Quantification of SHP2 staining intensity $(n=5)$ and of SHP2-positive cells $(n=5)$. d, e The mRNA $(n=5)(\mathbf{d})$ and protein level $(n=4)(\mathbf{e})$ of SHP2 are decreased in cultured SSc fibroblasts. Horizontal scale bar, for all images, $500 \mu \mathrm{m}$. All data are presented as median \pm s.e.m. The $p$ values are expressed as follows: $0.05>p>0.01^{\star} ; 0.01>p>0.001^{\star \star} ; p<0.001^{\star \star \star}$. Significance was determined by Mann-Whitney test. SSc: systemic sclerosis, Healthy: healthy individual, int.: intensity 
between $30 \mathrm{~min}$ and $6 \mathrm{~h}$. The less pronounced upregulation of SHP2 phosphatase activity by TGF $\beta$ at later time points parallels the inhibitory effects of TGF $\beta$ on SHP2 expression and may thus result from reduced SHP2 levels. Activation of TGF $\beta$ also stimulated Shp2 activity in vivo as demonstrated by increased Shp2 activity in the skin of mice overexpressing TBRICA as compared to control mice (Fig. 2i).

Shp2 regulates TGF $\beta$-induced fibroblast activation. To investigate the functional effects of decreased Shp2 levels, we knocked out $\operatorname{Sh} p 2$ by infecting dermal fibroblasts from $S h p 2^{f l / f l}$ mice with AdCre (Fig. 3a). Knockout of Shp2 ameliorated TGF $\beta$-induced myofibroblast differentiation with reduced mRNA and protein levels of $\alpha$-smooth muscle actin ( $\alpha$-SMA) and impaired formation of stress fibers (Fig. 3b-e). The induction of Colla1 mRNA and of collagen protein by TGF $\beta$ was also reduced in Shp2-deficient fibroblasts (Fig. 3f, g).

Knockout of SHP2 ameliorates fibrosis. To generate mice with fibroblast-specific, tamoxifen-inducible deletion of $\operatorname{Sh} p 2$, we a

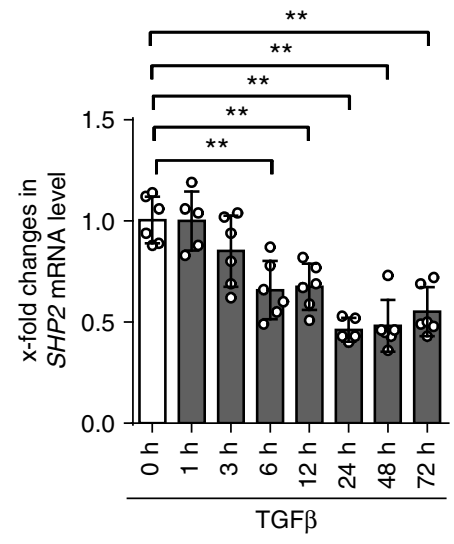

b
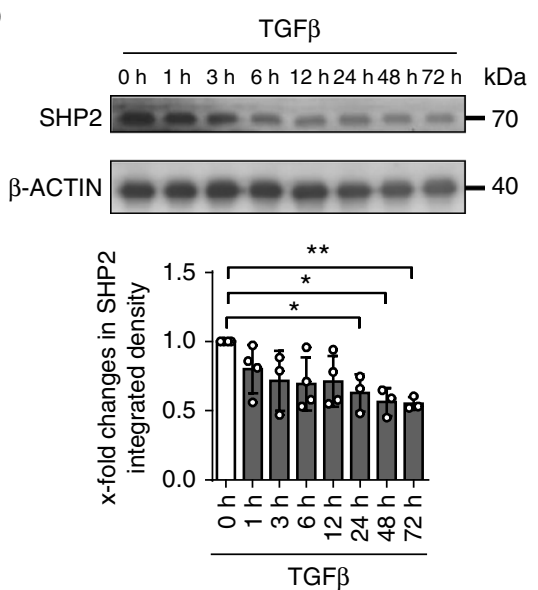

C

AdLacZ

$\mathrm{TBRI}^{\mathrm{CA}}$

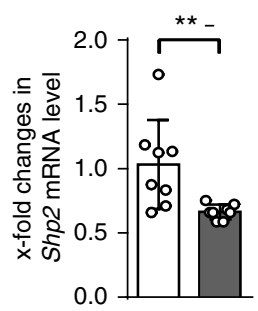

e

d

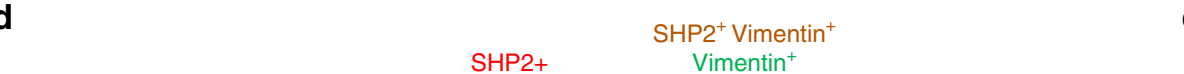

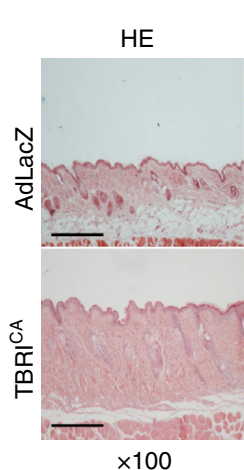

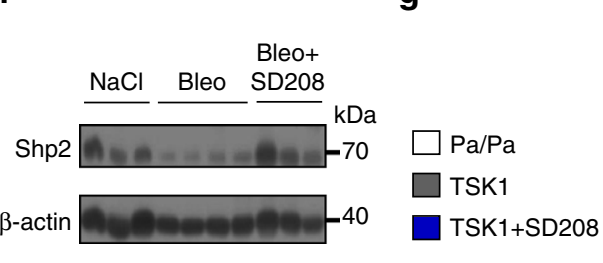

$\square \mathrm{NaCl}$

$\square$ Bleo

口 Bleo+SD208

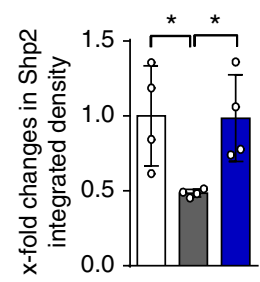

Vimentin
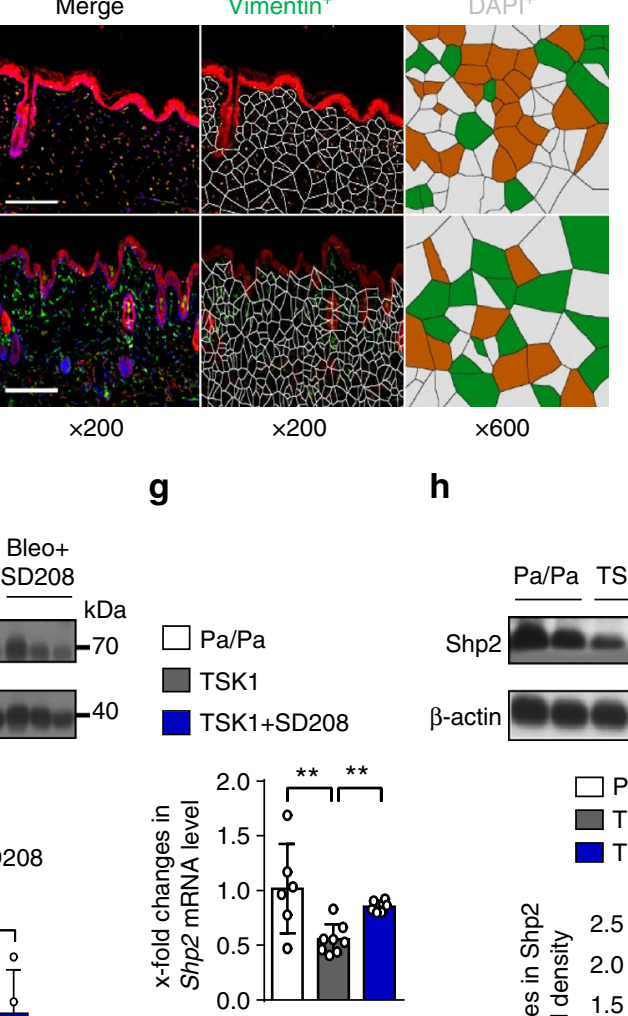

h

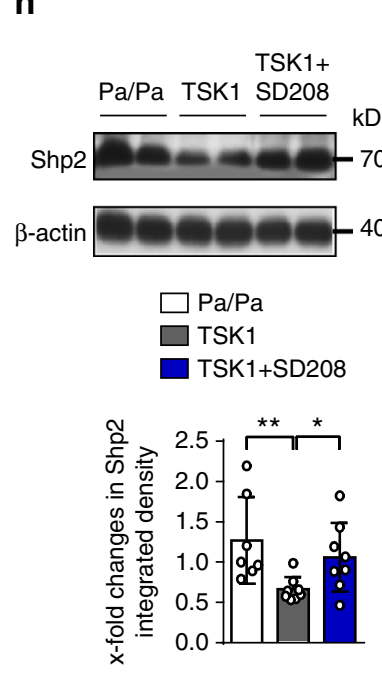

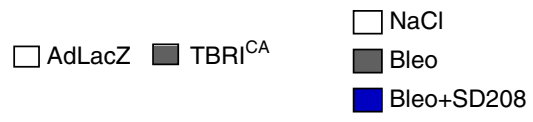
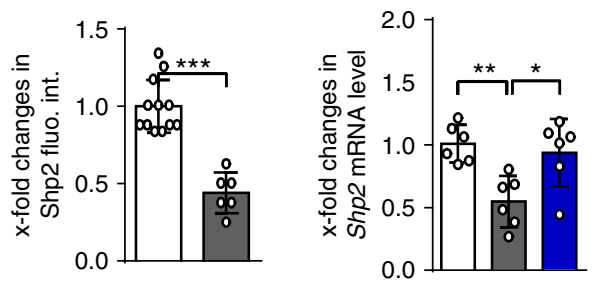

i
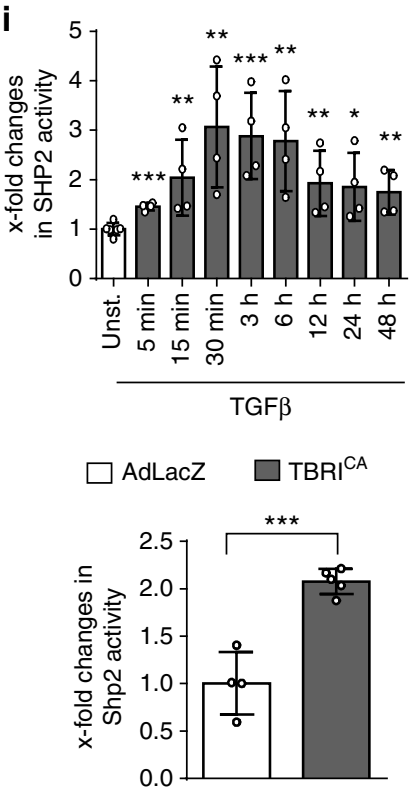
crossbred mice harboring Shp2 alleles flanked by loxP sites $\left(\operatorname{Shp} 2^{f l f l}\right)$ with mice expressing CreER under the control of a tamoxifen-inducible, fibroblast-specific type I collagen promoter $(\mathrm{Col1a2}-\mathrm{CreER})^{8}$. In the absence of fibrotic stimuli, mice with fibroblast-specific knockout of Shp2 (referred to as Shp2 Fib KO) did not show an overt phenotype and the skin architecture and the collagen content were comparable to Shp $2^{f l / f l_{X} \text { Colla2-CreER }}$ mice injected with corn oil (referred to as control mice).

However, $S h p 2$ Fib KO mice were protected from experimental skin fibrosis in different mouse models. Fibroblast-specific knockout of Shp2 ameliorated $\mathrm{TBRI}^{\mathrm{CA}}$-induced fibrosis with reduced dermal thickening, decreased myofibroblast counts and lower hydroxyproline content upon overexpression of TBRICA as compared to control mice (Fig. 4a). Shp2 Fib KO mice were also protected from bleomycin-induced skin fibrosis as an inflammation-driven model of fibrosis with decreased dermal thickening, impaired myofibroblast differentiation and reduced hydroxyproline content as compared to control littermates (Fig. 4b).

TSK1 mice represent a genetic model of fibrosis with endogenous, TGF $\beta$-dependent activation of fibroblasts. Fibroblast-specific knockout of $\operatorname{Sh} 22$ reduced the hypodermal thickening, the hydroxyproline content and the myofibroblast counts in the TSK1 model (Fig. 4c).

Knockout of Shp2 inhibits JAK2/STAT3 signaling. SHP2 has been shown to regulate angiotensin-II-induced activation of JAK/ STAT signaling in vascular smooth muscle cells ${ }^{4}$, mesangial cells $^{23}$ and hepatocellular carcinoma cells ${ }^{24}$. The effects of SHP2 on JAK/STAT have been shown to be highly context and/or cell type dependent: while SHP2 inhibits JAK/STAT signaling in leukocytes under inflammatory conditions, e.g., upon stimulation with IL- $6^{13,25}$, it promotes JAK/STAT signaling in mesenchymal cells under non-inflammatory conditions, e.g., upon stimulation with angiotensin ${ }^{26,27}$. JAK2 and STAT3 have recently been identified as downstream mediators of TGF $\beta$ signaling in fibro$\mathrm{sis}^{28-30}$. We therefore aimed to investigate whether SHP2 may modulate TGF $\beta$ signaling by regulation of JAK2/ STAT3 signaling. JAK2 activation was assessed by three approaches. First, by analysis of the phosphorylation status of JAK2 at Y1007/Y1008 (pJAK2 $\mathrm{Y}^{\mathrm{Y} 007 / \mathrm{Y} 1008}$ ), as phosphorylation of JAK2 at this particular site is considered as a key step in the activation of JAK2. As a second readout, we analyzed phosphorylation of STAT3 at Y705 (pSTAT3 ${ }^{\mathrm{Y705}}$ ), as STAT3 is a main downstream target of JAK2. Finally, we quantified changes in STAT3-dependent transcription in reporter studies. Knockout of Shp2 in cultured fibroblasts inhibited TGF $\beta$-induced JAK2/ STAT3 signaling with reduced levels of pJAK2 $\mathrm{Y}^{1007 / \mathrm{Y} 1008}$ and decreased levels of pSTAT3 (Fig. 5a). The total expression levels of JAK2 and STAT3 did not change. Knockdown of SHP2 also ameliorated STAT3-dependent reporter activity (Fig. 5a). The impaired activation of JAK2/STAT3 signaling upon inactivation of Shp2 was confirmed in experimental fibrosis. Fibroblastspecific knockout of $\operatorname{Sh} p 2$ was associated with decreased levels of pJAK2 ${ }^{\mathrm{Y} 1007 / \mathrm{Y} 1008}$ and pSTAT3 ${ }^{\mathrm{Y} 05}$ in TBRICA (Fig. 5b) and bleomycin-induced fibrosis (Fig. 5c) as well as in TSK1 mice (Fig. 5d) compared to corresponding controls.

SHP2 regulates TGF $\beta$ signaling via its phosphatase activity. SHP2 can regulate growth factor signaling by dephosphorylation of target proteins or in a phosphatase-independent manner ${ }^{18}$. To investigate whether the phosphatase activity of SHP2 is required for regulation of TGF $\beta$ signaling in fibroblasts, we overexpressed a phosphatase-dead mutant of SHP2 (SHP2 ${ }^{\mathrm{C} 459 \mathrm{~S}}$ ) in cultured human fibroblasts and compared the effects of overexpression of $\mathrm{SHP}_{2}{ }^{\mathrm{C}} 459 \mathrm{~S}$ and the non-mutated SHP2 (SHP2 ${ }^{\mathrm{WT}}$ ) on TGF $\beta$ signaling and fibroblast activation. Overexpression of $\mathrm{SHP}_{2} \mathrm{WT}$ increased the mRNA levels of COL1A1 and ACTA2, increased the release of collagen (Fig. 6a, b) and upregulated the levels of $a$ SMA and the formation of stress fibers (Fig. $6 \mathrm{c}, \mathrm{d}$ ) as compared to control cells transfected with the empty coding vector. In contrast, overexpression of $\mathrm{SHP}_{2} \mathrm{C} 459 \mathrm{~S}$ did not enhance fibroblast activation, but rather acted in a dominant negative manner to suppress TGF $\beta$-induced fibroblast activation (Fig. 6c, d). Consistently, JAK2/STAT3 signaling was enhanced by overexpression of SHP2 ${ }^{\mathrm{WT}}$ with decreased levels of $\mathrm{pJAK} 2 \mathrm{Y}^{\mathrm{Y} 70}$ and increased levels of pJAK2 ${ }^{\mathrm{Y} 1007 / \mathrm{Y} 1008}$ and pSTAT3 ${ }^{\mathrm{Y} 705}$, while it was found suppressed by overexpression of SHP2 ${ }^{\mathrm{C} 459 \mathrm{~S}}$ (Fig. 6b).

To further confirm that SHP2 regulates TGF $\beta$-induced JAK2/ STAT3 signaling by dephosphorylation of JAK2 at the inhibitory phosphorylation site at Y570, we overexpressed a mutant JAK2 resistant to phosphorylation at $\mathrm{Y} 570\left(\mathrm{JAK} 2^{\Delta \mathrm{Y} 570 \mathrm{~F}}\right)$ in fibroblasts.

Overexpression of JAK $2^{\triangle \mathrm{Y} 570 \mathrm{~F}}$ promoted activation of resting fibroblasts and rendered them more susceptible to the stimulatory effects of TGF $\beta$ as compared to fibroblasts transfected with control vector or non-mutated JAK2 vectors (Fig. 7a-c). Treatment with NSC-87877, an inhibitor of both SHP1 and SHP2, ameliorated the stimulatory effects of TGF $\beta$ in fibroblasts transfected with control vector or with non-mutated JAK2 constructs. However, fibroblasts overexpressing JAK2 ${ }^{\triangle \mathrm{Y} 570 \mathrm{~F}}$ were insensitive to SHP2 inhibition. Treatment with NSC-87877 did not decrease the mRNA levels of COL1A1 and COL1A2, the release of collagen protein, $\alpha$-SMA expression and stress fiber formation in fibroblasts overexpressing JAK2 ${ }^{\triangle \mathrm{Y} 570 \mathrm{~F}}$ (Fig. 7a-c). Similar findings were obtained in STAT3 reporter assays (Fig. 7d). Overexpression of JAK2 $2^{\triangle \mathrm{Y} 570 \mathrm{~F}}$ increased STAT3 reporter activity and cells overexpressing $\mathrm{JAK} 2^{\triangle \mathrm{Y} 570 \mathrm{~F}}$ were insensitive to the inhibitory effects of SHP2 inhibition on STAT3 reporter activity. We next aimed to show that TGF $\beta$ promotes binding of SHP2 to JAK2. Indeed, stimulation of fibroblasts with TGF $\beta$ promoted interaction of SHP2 with JAK2 and increased amounts of JAK2 precipitated with SHP2 in fibroblasts upon stimulation with TGF $\beta$ (Fig. 7e). Together, these data demonstrate that TGF $\beta$

Fig. 2 SHP2 is downregulated in TGF $\beta$ signaling. a, b Decreased mRNA $(n=6)(\mathbf{a})$ and protein $(n=4)(\mathbf{b})$ levels of SHP2 in healthy fibroblasts stimulated with TGF $\beta(10 \mathrm{ng} / \mathrm{ml})$ for different time points as measured by RT-PCR and western blot, respectively. c, d Overexpression of TBRICA $\left(6.67 \times 10^{7}\right.$ IFUs every 2 weeks) significantly reduced mRNA $(n=8)$ and the protein levels of Shp2 in murine skin as shown by qPCR (c) and immunofluorescence staining (d) of Shp2 with co-staining for fibroblast marker Vimentin and DAPI ( $n \geq 6$ per each group). Representative images are shown at 100-200- and 600-fold magnification. Horizontal scale bar, $500 \mu \mathrm{m}$. Immunofluorescence pictures were analyzed by Voronoi tessellation. e, f Treatment with the selective TGF $\beta$ receptor type 1 kinase inhibitor SD208 $(60 \mathrm{mg} / \mathrm{kg} /$ day) reversed the decrease of Shp2 mRNA $(n=6)(\mathbf{e})$ and protein $(n=4)(\mathbf{f})$ in bleomycin-challenged mice ( $50 \mu$ g every other day). $\mathbf{g}$, $\mathbf{h}$ Treatment with the selective TGF $\beta$ receptor type 1 kinase inhibitor SD-208 reversed the decrease of Shp2 mRNA ( $n=6$ ) (g) and protein (h) in TSK1 mice ( 2 mg tamoxifen over 5 days) ( $n \geq 6$ per each group). i Phosphatase activity assay. Increases in SHP2 activity after TGF $\beta$ stimulation $(10 \mathrm{ng} / \mathrm{ml})(n=4)$ in cultured fibroblasts and upon overexpression of TGF $\beta R \mathrm{RI}\left(6.67 \times 10^{7} \mathrm{IFUs}\right)$ in murine skin ( $n \geq 4$ per each group). Results shown are representative of three independent experiments. All data are presented as median \pm s.e.m. The $p$ values are expressed as follows: $0.05>p>$ $0.01^{\star} ; 0.01>p>0.001^{\star \star} ; p<0.001^{\star \star \star}$. Significance was determined by Mann-Whitney test. AdLacZ: adenovirus LacZ, TBRICA: constitutively active TGF $\beta$ receptor type I, TSK1: Tight skin, Bleo: bleomycin, Pa/Pa: control for TSK1, fluo.: fluorescence, int.: intensity, Unst.: unstimulated 
a

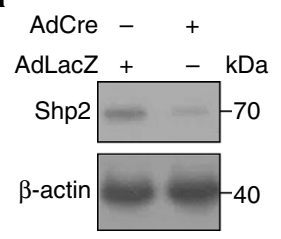

$\square$ AdLacZ $\square$ AdCre

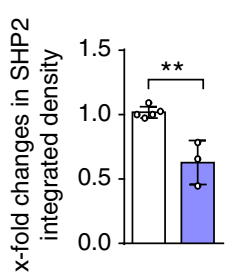

d
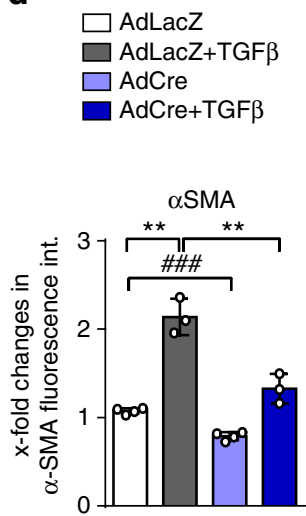

b

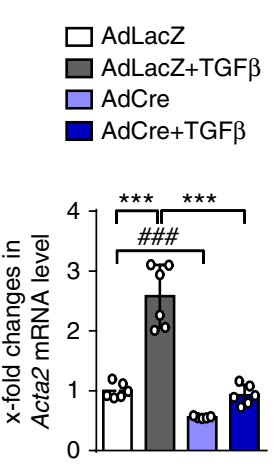

e

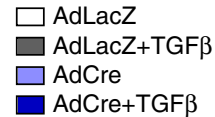

Stress fibers

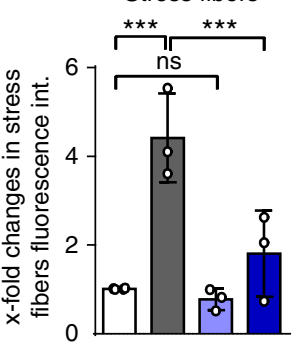

C

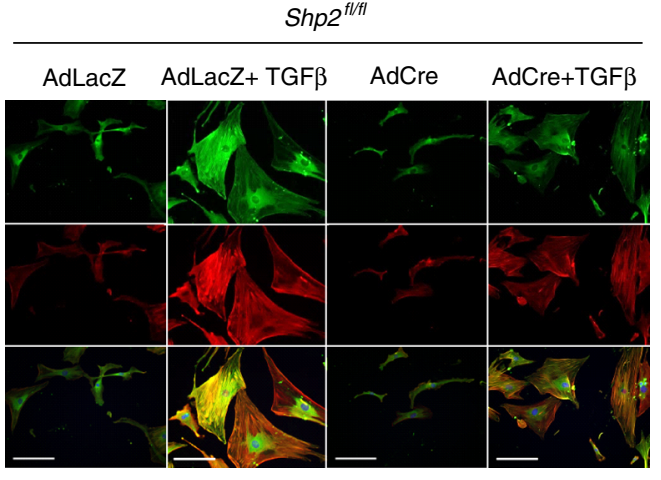

f

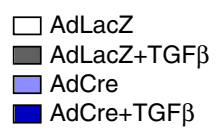

g

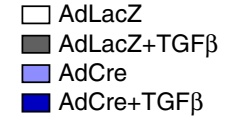

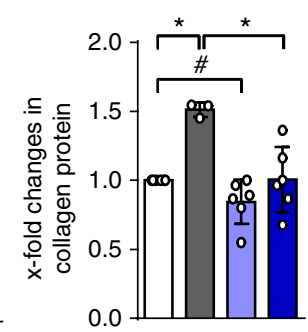

Fig. 3 Shp2 regulates TGF $\beta$ induced fibroblast activation. a Western blot for efficiency of Cre-mediated (80 IFUs/cell) knockout of Shp2 in murine Shp2 $2^{f l / f l}$ fibroblasts ( $n \geq 3$ per each group). b Shp2 knockout decreased mRNA levels of Acta2 $(n=6)$. c-e Shp2 knockout decreased $\alpha$-SMA and stress fiber staining. Representative images are shown at 200-fold magnification (c). Horizontal scale bar, $500 \mu \mathrm{m}$. Quantification of $\alpha$-SMA staining intensity (d) and stress fiber staining intensity (e) ( $n \geq 3$ different lines). f, g Col1a1 mRNA (f) and collagen protein release (g) induced by TGF $\beta$ ( $10 \mathrm{ng} / \mathrm{ml}$ for $24 \mathrm{~h}$ ) ( $n \geq 3$ different lines). Results shown are representative of three independent experiments. All data are presented as median \pm s.e.m. The $p$ values are expressed as follows: $0.05>p>0.01^{\star}$ or $\# ; 0.01>p>0.001^{\star \star} ; p<0.001^{\star \star \star}$ or \#\#; ns: not significant. Significance was determined by Mann-Whitney test. AdCre: adenovirus Cre, AdLacZ: adenovirus LacZ, int.: intensity

induces SHP2-dependent dephosphorylation of JAK2 at Y570 to promote activation of STAT3.

As NSC-87877 does not discriminate between SHP1 and SHP2 and may also inhibit other tyrosine phosphatases in higher concentrations (half-maximal inhibitory concentration $\left(\mathrm{IC}_{50}\right)$ for SHP2 is $0.318 \mu \mathrm{M}$, for SHP1 $0.355 \mu \mathrm{M}$, for PTP $1 \mathrm{~b}=1.691 \mu \mathrm{M}$ and for $\mathrm{HePTP}=7.745 \mu \mathrm{M}$ ), we aimed to confirm our findings with more specific SHP2 inhibitors. To better discriminate between SHP1- and SHP2-mediated effects, we confirmed our results with SHP099 (allosteric inhibitor) and 11-al (active site inhibitor), both of which possess high selectivity for SHP2 over SHP $1^{20,31,32}$. Both inhibitors effectively reduced TGF $\beta$-induced fibroblast activation to an extent similar to that observed with NSC-87877 (Supplementary Fig. 2a-c).

Inhibition of SHP2 exerts anti-fibrotic effects. After demonstrating that fibroblast-specific genetic inactivation of Shp2 ameliorates experimental fibrosis, we next aimed to investigate the anti-fibrotic potential of pharmacological inhibition of SHP2. Incubation with NSC-87877 ameliorated the stimulatory effects of TGF $\beta$ on COL1A1 mRNA and release of collagen protein (Fig. 8a) and inhibited myofibroblast differentiation with reduced mRNA (Fig. 8b) and protein levels of a-SMA and impaired formation of stress fibers (Fig. 7c) at non-toxic concentrations (Supplementary Fig. 3a). In accordance with our proposed mode of action
(Supplementary Fig. 4a-b), incubation with NSC-87877 inhibited the accumulation of pJAK2 $2^{\mathrm{Y} 1007 / \mathrm{Y} 1008}$ and of its downstream target pSTAT3, but increased the levels of pJAK2 $2^{\mathrm{Y} 570}$ in TGF $\beta$ stimulated fibroblasts (Fig. 8d). Consistently, incubation with NSC-87877 inhibited the TGF $\beta$-induced activation of STAT3dependent transcription in reporter assays (Fig. 8e).

Treatment with NSC-87877 also ameliorated bleomycininduced skin fibrosis with decreased dermal thickening, hydroxyproline content and myofibroblast counts as compared to vehicle-treated mice (Fig. 9a). Similar results were obtained in the $\mathrm{TBRI}^{\mathrm{CA}}$-induced skin fibrosis (Fig. 9b). In addition, NSC87877 also ameliorated bleomycin-induced pulmonary fibrosis (Fig. 9c).

We employed three different inhibitors of SHP2, the active site inhibitors 11-a1 and PHPS1, as well as the allosteric inhibitor SHP09931-33 to confirm the findings obtained with the SHP1/ SHP2 inhibitor NSC-87877. This may be of particular importance as SHP1 has been shown to inhibit proliferation of pro-fibrotic hepatic stellate cells and may thus also modulate the outcome of fibrotic diseases ${ }^{34,35}$. 11-a1, PHPS1 and SHP099 all ameliorated bleomycin-induced pulmonary fibrosis (Fig. 10a) and TBRICA induced dermal fibrosis (Fig. 10b). Hydroxyproline content, myofibroblast counts and fibrotic area or dermal thickness, respectively, were significantly reduced in mice treated with 11a1, PHPS1 or SHP099 compared to vehicle-treated controls and 
a
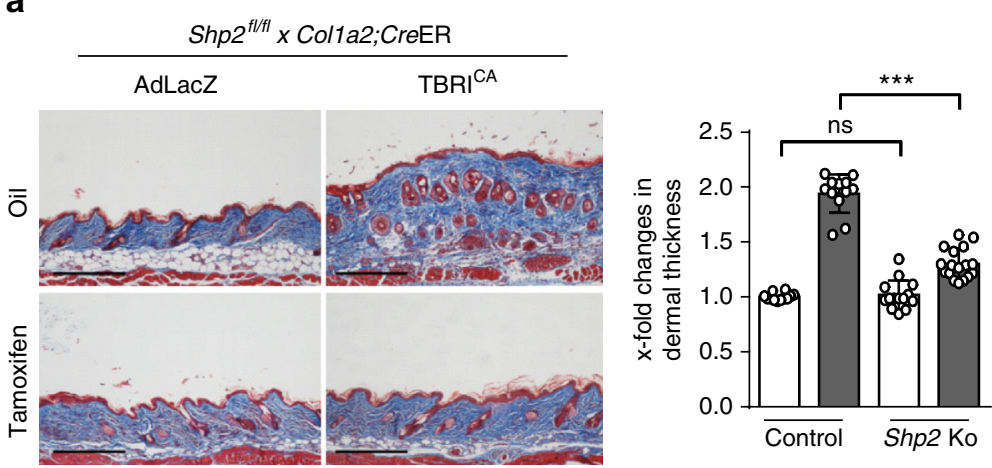

AdLacZ

$\mathrm{TBRI}^{\mathrm{CA}}$

b
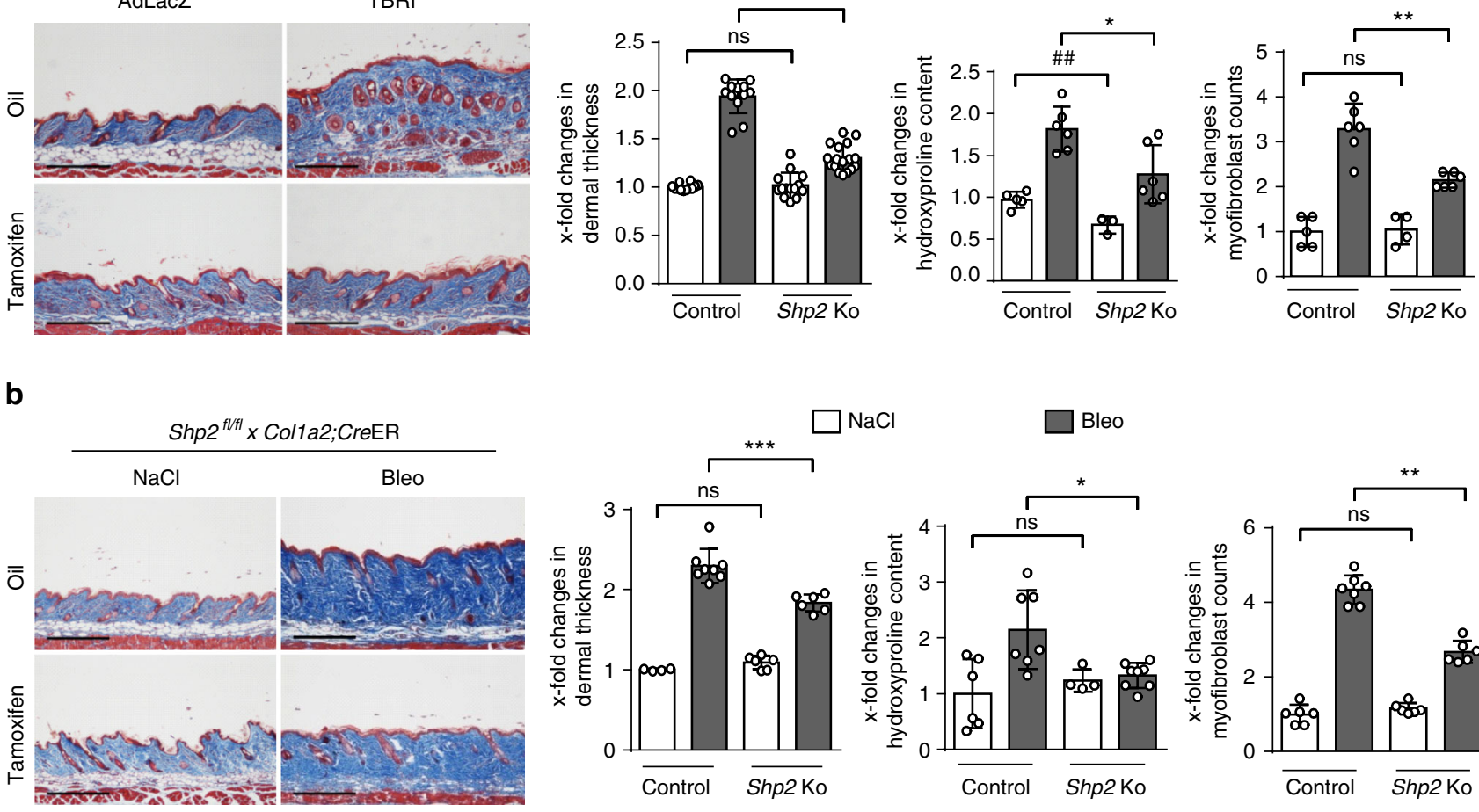
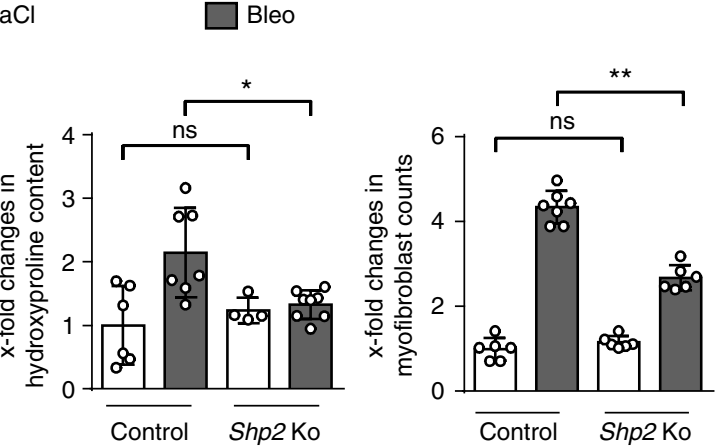

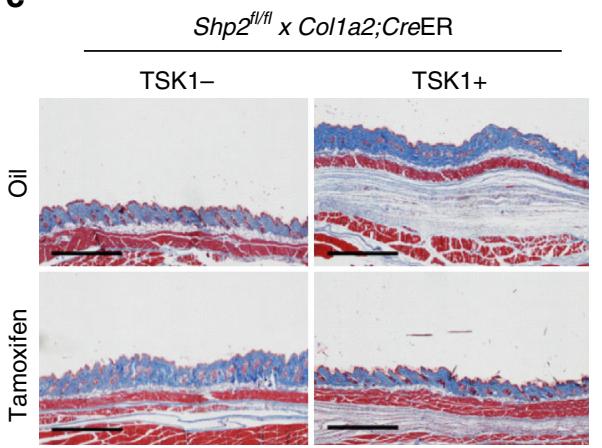

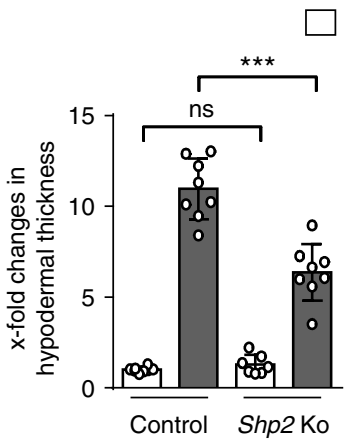

Fig. 4 Fibroblast-specific knockout of Shp2 protects from experimental fibrosis. a TBRICA-induced fibrosis ( $6.67 \times 10^{7}$ IFUs every 2 weeks). Representative images of Masson trichrome-stained skin shown at 100-fold magnification. Dermal thickness, hydroxyproline content and myofibroblast counts. All groups consisted of $\geq 9$ mice each. b Bleomycin-induced skin ( $50 \mu \mathrm{g}$ every other day) fibrosis. Representative images of Masson trichrome-stained skin shown at 100 -fold magnification. Dermal thickness, hydroxyproline content and myofibroblast counts. All groups consisted of $\geq 8$ mice each. c TSK1 model (2 mg tamoxifen over 5 days). Representative images of Masson trichrome-stained skin shown at 40-fold magnification. Hypodermal thickness, hydroxyproline content and myofibroblast counts. All groups consisted of $\geq 8$ mice each. Horizontal scale bar in all images, $500 \mu \mathrm{m}$. All data are presented as median \pm s.e. m. The $p$ values are expressed as follows: $0.05>p>0.01^{\star} ; 0.01>p>0.001^{\star \star}$ or \#\#; $p<0.001^{\star \star \star}$; ns: not significant. AdLacZ: adenovirus LacZ, TBRICA: constitutively active TGF $\beta$ receptor type I, TSK1: Tight skin, Bleo: bleomycin, Shp2 Ko: SHP2 fibroblast-specific knockout

the anti-fibrotic effects were in the range of those observed with NSC-87877 in previous experiments.

\section{Discussion}

Our data characterize SHP2 as an important regulator of TGF $\beta$ signaling in fibroblasts. Shp2-deficient murine fibroblasts are less responsive to TGF $\beta$. An impaired response to the pro-fibrotic effects of TGF $\beta$ was also observed in human fibroblasts upon pharmacological inhibition of SHP2. Consistent with these findings, inhibition of SHP2 prevented epithelial-to-mesenchymal transition in A549 adenocarcinoma cells ${ }^{36}$. In contrast, ectopic expression of full-length but not of phosphatase-deficient SHP2 in fibroblasts enhances TGF $\beta$ signaling. Mechanistically, SHP2 regulates the TGF $\beta$-dependent activation of JAK2. The tyrosine kinase JAK2 has recently been identified as a pro-fibrotic mediator. Inhibition of JAK2 exerts anti-fibrotic effects and has been shown to ameliorate skin, liver, pulmonary and renal fibrosis $^{29,37,38}$. The activity of JAK2 is tightly regulated by phosphorylation. Depending on the actual target site, these phosphorylation events can either activate or inhibit JAK2. Phosphorylation of JAK2 at Y570 inhibits JAK2 activation. Dephosphorylation of JAK2 at Y570 is a pre-requisite for JAK2 to undergo the activating phosphorylation at Y1007/Y1008, whereas mutation of the Y570 leads to enhanced and prolonged JAK2 activation $^{39}$. We demonstrate that SHP2 can dephosphorylate JAK2 at Y570 to promote TGF $\beta$-dependent activation of JAK2 and its downstream mediator STAT3 (Supplementary Fig. 4a). In contrast, pharmacological inhibition of SHP2, overexpression of a 
a
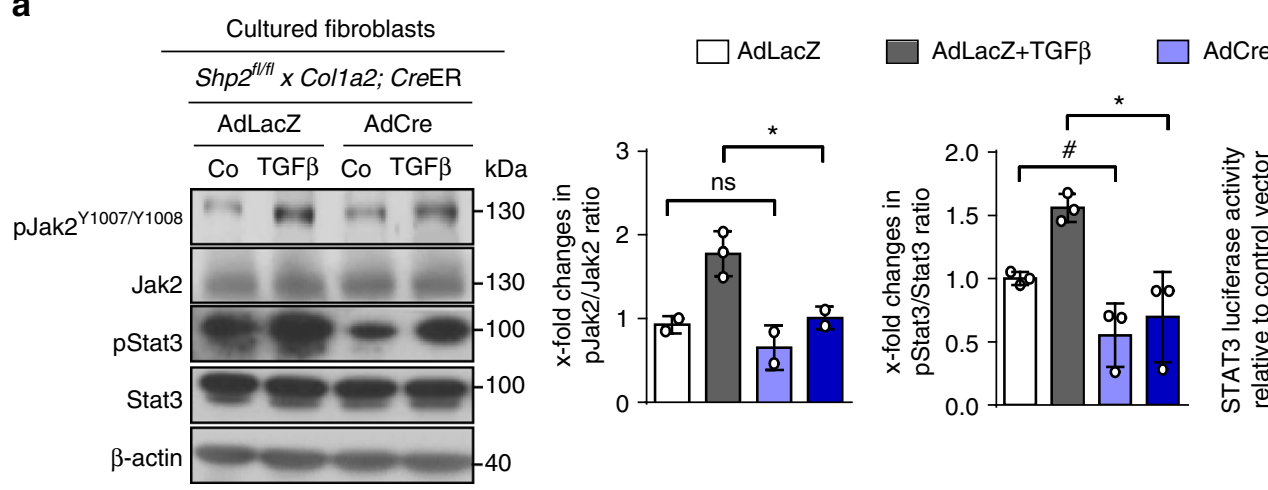

AdCre+TGF $\beta$

b
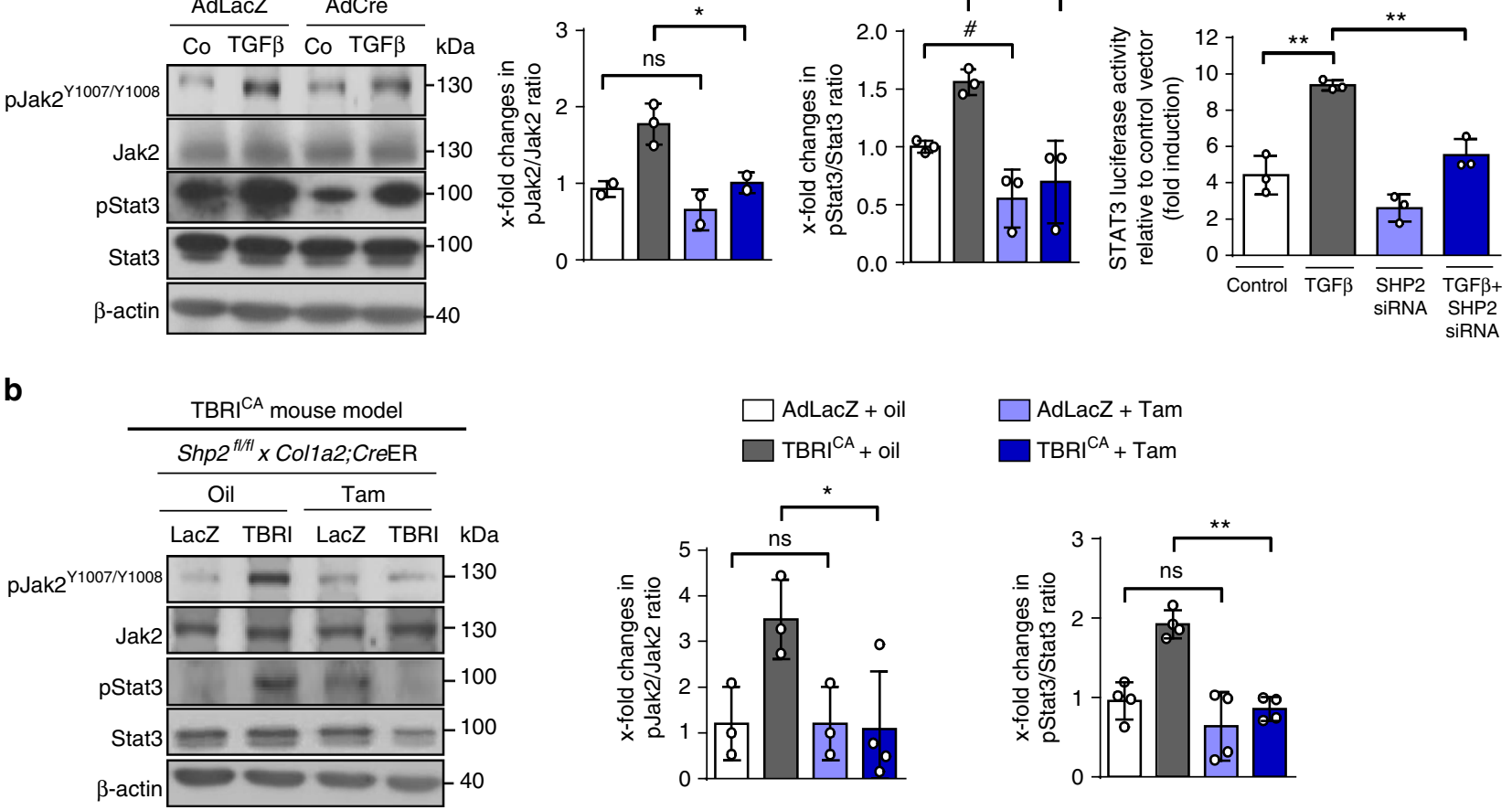

C
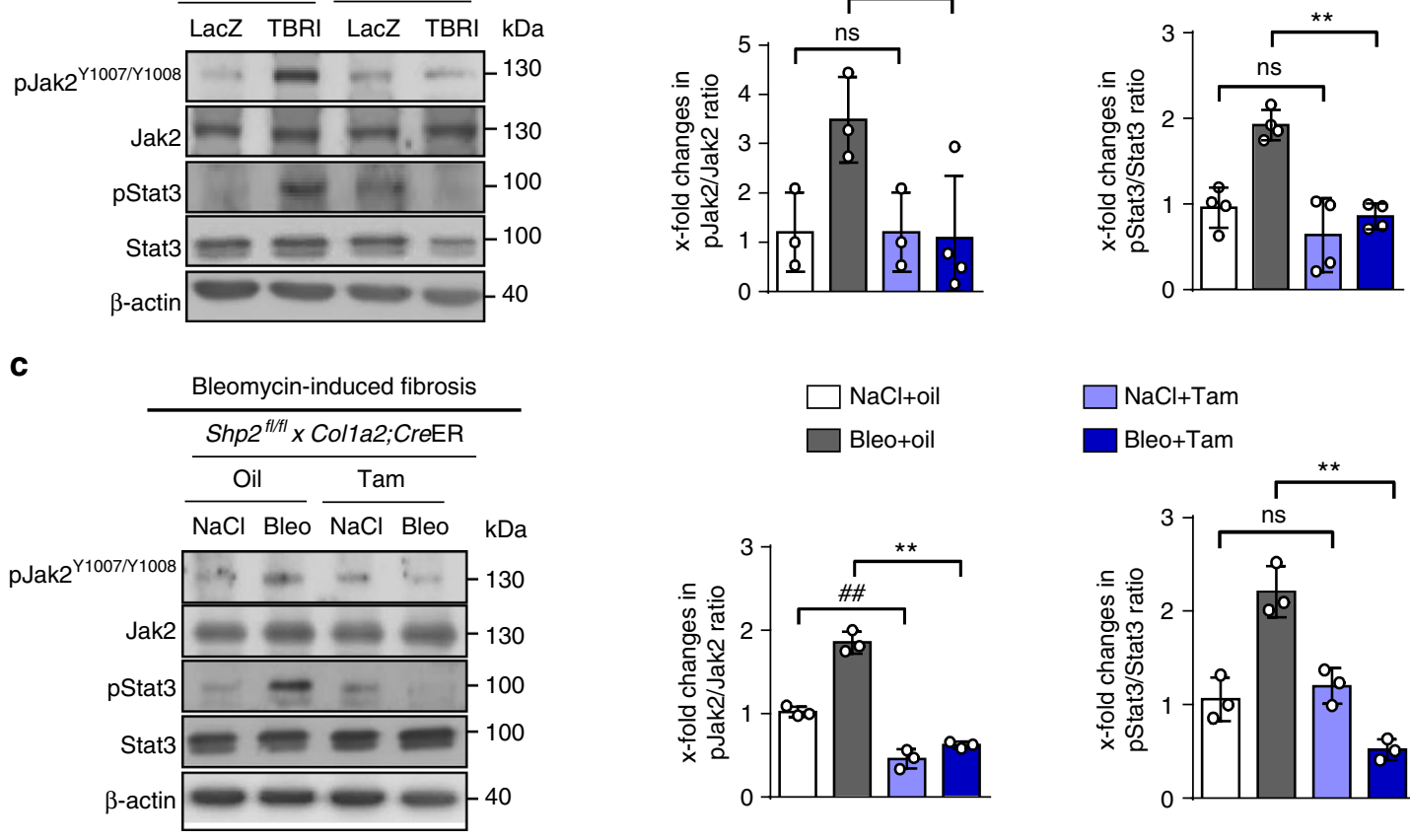

d
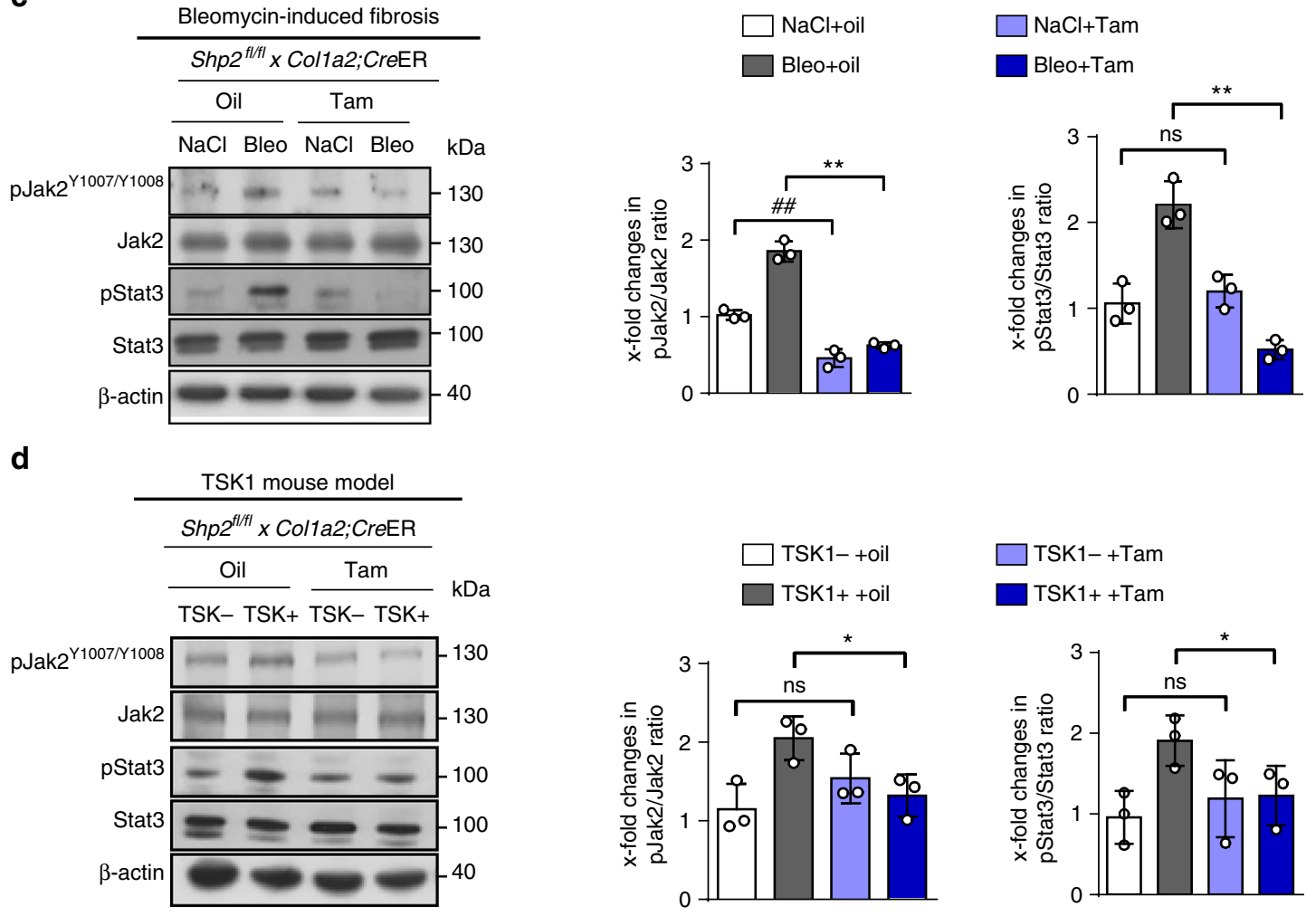

Fig. 5 Knockout of Shp2 decreases JAK2/STAT3 signaling. a Knockout of Shp2 in fibroblasts (Shp2fl/fl $x$ Colla2; CreER) decreases the levels of pJAK2 and pSTAT3 and STAT3 reporter activity in cultured fibroblasts ( $n=3$ different lines). Cells were stimulated with TGF $\beta$ ( $10 \mathrm{ng} / \mathrm{ml}$ for $6 \mathrm{~h}$ ). b-d Conditional knockout of Shp2 reduces the levels of pJAK2 and pSTAT3 in TBRICA (6.67 $\times 10^{7}$ IFUs every 2 weeks) (b) and bleomycin-induced fibrosis (50 $\mu$ g every other day) (c) and in TSK1 mice (2 mg tamoxifen over 5 days) (d) ( $n \geq 3)$. Results shown are representative of three independent experiments. All data are presented as median \pm s.e.m. The $p$ values are expressed as follows: $0.05>p>0.01^{\star}$ or $\# ; 0.01>p>0.001^{\star \star}$ or $\#$; $p<0.001^{\star \star *}$; ns: not significant. Significance was determined by Mann-Whitney test. AdLacZ or LacZ: adenovirus LacZ, TBRICA or TBRI: constitutively active TGF $\beta$ receptor type I, TSK1: Tight skin, Bleo: bleomycin, Tam: tamoxifen, Co: Control unstimulated, AdCre: adenovirus Cre 
catalytically inactive SHP2 mutant or knockout of Shp2 inhibits dephosphorylation of $\mathrm{pJAK2} 2^{\mathrm{Y} 570}$, which prevents phosphorylation of JAK2 at Y1007/Y1008 and subsequent activation of STAT3 in TGF $\beta$-stimulated fibroblasts and in experimental fibrosis $^{30,40}$.

We previously characterized the serine/threonine kinase CK2 as an upstream activator of JAK2/STAT3 signaling in fibroblasts.
Pharmacological inhibition of CK2 ameliorated experimental fibrosis and those anti-fibrotic effects were associated with decreased levels of pJAK2 $2^{\mathrm{Y} 1007 / 1008}$ and $\mathrm{pSTAT}^{40}$. However, SHP2 regulates JAK2 signaling at a different level and by a different mechanism than CK2. Although the precise mechanisms by which the serine/threonine kinase CK2 promotes accumulation of pJAK2 $2^{\mathrm{Y} 1007 / 1008}$ and pSTAT3 have not been uncovered, a

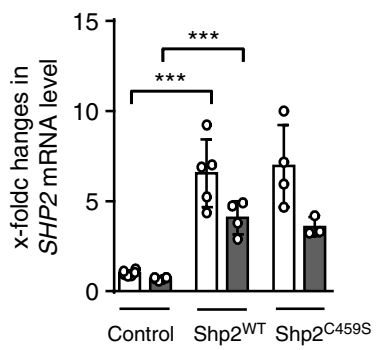

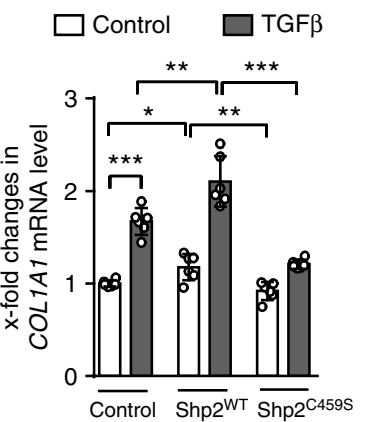

b

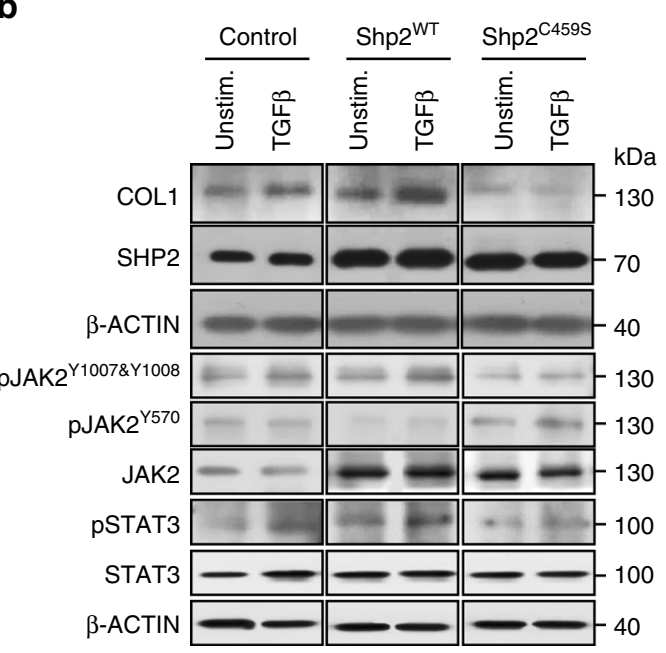

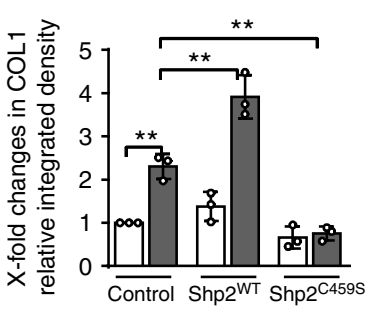
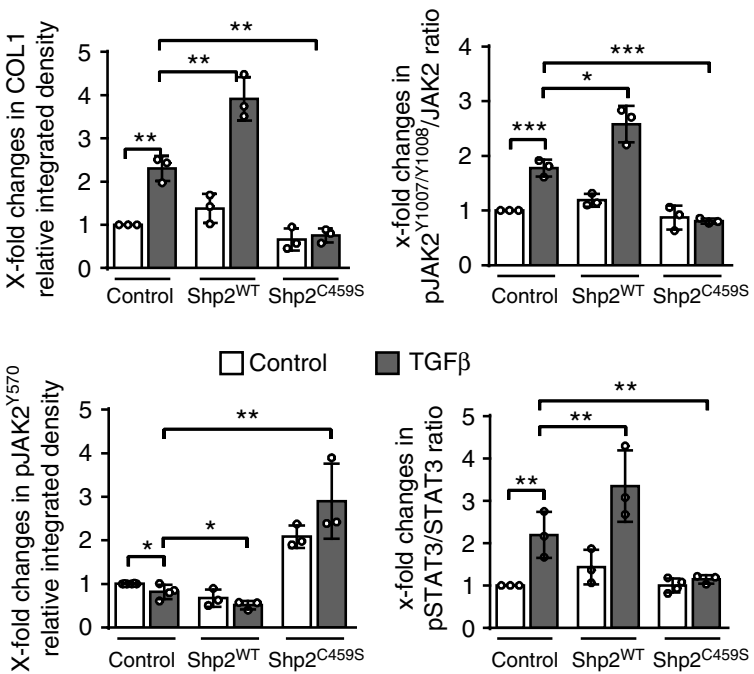

C

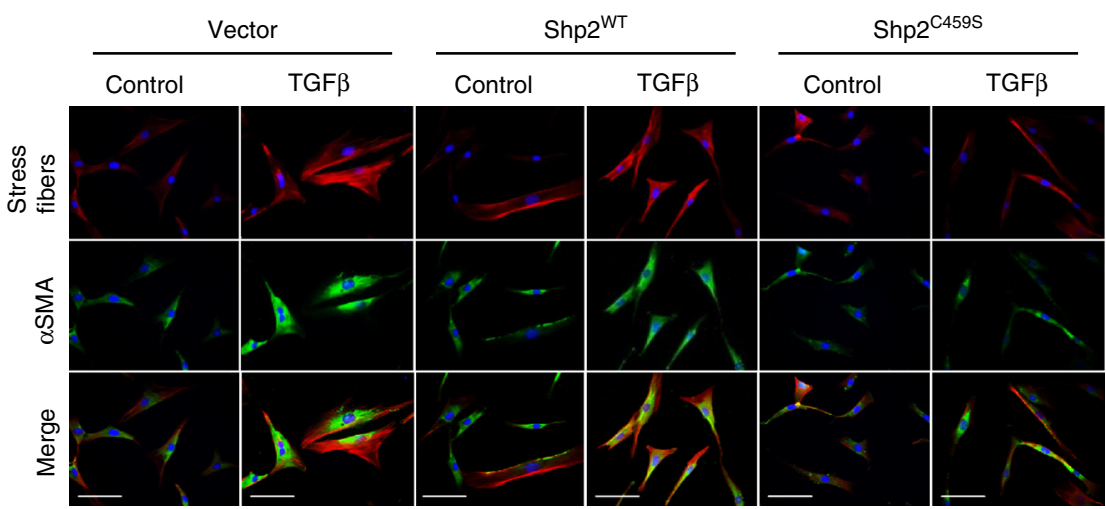

d

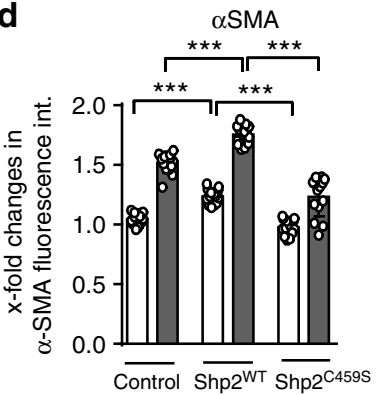

Stress fibers

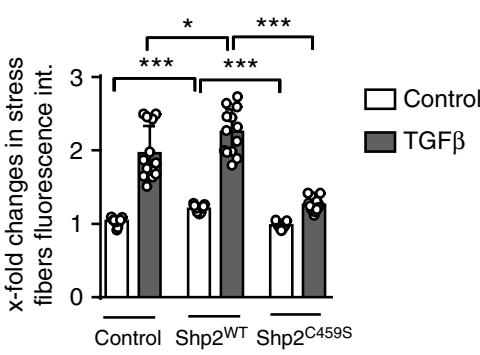


the effects seem to be indirect, given the delayed effects of CK2 on JAK2 signaling. CK2 may thus serve to amplify JAK2 signaling by enhancing positive signaling input. In contrast, SHP2 provides a permissive signaling environment, as it removes the inhibitory phosphorylation of JAK2 at Y570, which is required for subsequent phosphorylation at Y1007/1008 and thus for activation of JAK2 signaling. SHP2-induced removal of this inhibitory phosphorylation mark may thus be a pre-requisite for effective activation of JAK2 signaling, which may explain the potent antifibrotic effects of targeting SHP2.

The activating effects of SHP2 on TGF $\beta$-induced JAK2/STAT3 activation directly translate into stimulatory effects on fibroblasts. Fibroblasts overexpressing SHP2 are more susceptible to the profibrotic effects of TGF $\beta$, whereas the stimulatory effects of TGF $\beta$ on myofibroblast differentiation and collagen release are reduced in $\operatorname{Shp} 2$ knockout fibroblasts. Selective inactivation of $\operatorname{Sh} p 2$ in fibroblasts also reduced the pro-fibrotic effects of TGF $\beta$ signaling in vivo. Shp2 Fib KO mice were protected from experimental fibrosis induced by overexpression of a constitutively active TGF $\beta$ receptor type I. Moreover, fibroblast-specific inactivation of Shp2 also protected from bleomycin-induced skin fibrosis and ameliorated fibrosis in TSK1 mice, thereby confirming the central regulatory function of Shp2 on TGF $\beta$ signaling and fibroblast activation in multiple complementary models of SSc. Consistent with the role of SHP2 as a mediator of tissue remodeling, inactivation of $\operatorname{Shp} 2$ in airway epithelial cell reduced pulmonary remodeling in response to ovalbumin challenge as a model of chronic asthma ${ }^{41}$. Our findings are also in line with recent results which show that SHP2 is required for epithelial-to-mesenchymal transition induced by IL- 6 in breast cancer cells ${ }^{42}$. Our findings may also be supported by reports about fibrotic changes in patients with Noonan syndrome with hyperactive SHP2 such as myocardial fibrosis ${ }^{43,44}$, fibrosis of the extraocular muscles ${ }^{45}$ and recurrent keloid formation ${ }^{34}$.

The inhibitory effects of SHP2 on fibroblast activation may not be limited to myofibroblasts. Inhibition of SHP2 has recently been shown to ameliorate the responsiveness of synovial fibroblast-like cells from patients with rheumatoid arthritis that exhibit a characteristic inflammatory and invasive phenotype to tumor necrosis factor and PDGF 21,22 . Together, these findings identify SHP2 as a key regulator of growth factor-induced fibroblast activation.

These findings may have translational implications. The critical role of SHP2 in various types of cancer prompted the development of small inhibitors of $\mathrm{SHP} 2^{31}$, some of which already showed promising results in first clinical trials ${ }^{46}$. The SHP inhibitor NSC-87877, which serves as a lead compound for the development of new SHP inhibitors, exerted anti-fibrotic effects

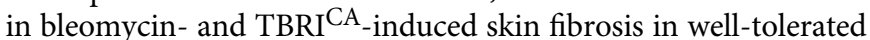
doses. These murine models resemble different stages and subgroups of SSc. The mouse model of bleomycin-induced dermal fibrosis mimics inflammatory stages of SSc, in which fibroblasts are pre-dominantly activated by pro-fibrotic mediators released from infiltrating leukocytes. In contrast, the mouse model of
$\mathrm{TBRI}^{\mathrm{CA}}$-induced fibrosis resembles $\mathrm{SSc}$ patients, in which inflammatory infiltrates have largely resolved and fibroblasts are endogenously activated ${ }^{47}$. Moreover, treatment with NSC-87877 also ameliorated pre-established bleomycin-induced pulmonary fibrosis as the leading cause of fibrosis-associated death in SSc. Extrapolating the findings from the mouse models to humans, these findings may indicate that SHP2 plays a crucial role in the pathogenesis of inflammatory as well as non-inflammatory types of fibrotic diseases, that the pro-fibrotic effects of SHP2 are not limited to the skin, but are also operative in the lung and that inhibition of SHP2 is not only effective in preventive, but also in therapeutic settings. However, considering the complex pathogenesis and the heterogeneity of SSc, further in vivo studies are required to confirm these findings. Particular attention should be appointed to the effects of SHP2 inhibition on macrophage polarization. A recent report demonstrated that SHP2 is required for M1 polarization of macrophages in the context of Haemophilus influenza infection ${ }^{48}$ and that inactivation of SHP2 may promote M2 polarization ${ }^{49}$. Macrophage polarization in SSc is skewed towards M2 polarization and those alternatively activated fibroblasts are thought to play an important role in fibroblast activation by the release of pro-fibrotic mediators such as IL-4 and IL-13 ${ }^{50,51}$. Indeed, selective knockout of SHP2 may actually promote experimental fibrosis ${ }^{49}$. Thus, a careful selection for patients with less inflammatory activity may be required to ensure that the beneficial effect of SHP2 inhibition on fibroblasts are not outweighed by the effects on M2 polarization ${ }^{52}$.

We focused on the role of SHP2 in the pathogenesis of fibrosis. However, SHP2 is also differentially expressed in microvascular endothelial cells of SSc patients as demonstrated by immunohistochemistry in our study and first evidence links SHP2 to the pathogenesis of vascular diseases. SHP2 has been reported to enhance PDGF signaling during vascular neointima formation ${ }^{53}$ and to be required for angiotensin-II-induced apoptosis of pulmonary endothelial cells ${ }^{54}$. The role of SHP2 in the vascular pathogenesis of SSc requires further investigation in murine models that resemble the vascular alterations in SSc such as Fra-2 transgenic mice or uPAR (urokinase-type plasminogen activator receptor) knockout mice ${ }^{55,56}$.

In summary, we characterize SHP2 as a positive regulator of TGF $\beta$-dependent activation of JAK2/STAT3 signaling. Genetic or pharmacologic inactivation of SHP2 inhibits JAK2/ STAT3 signaling, reduces fibroblast activation and ameliorates experimental fibrosis in several complementary models. These findings identify SHP2 as a potential molecular target for the treatment of fibrosis in fibrotic diseases such as SSc.

\section{Methods}

Patients. Dermal fibroblasts were isolated from skin biopsies of 24 SSc patients and 28 age- and sex-matched healthy volunteers. All patients fulfilled the ACR/EULAR (American College of Rheumatology/European League Against Rheumatism) 2013 criteria $^{57}$. Sixteen patients were female and seven were male. The median age of SSc patients was 49 years (range: 19-72 years), and their median disease duration was 6 years (range: 1-12 years). The human studies were approved by the Ethica committee of the Medical Faculty of the University of Erlangen-Nuremberg. All

Fig. 6 SHP2 enhances TGF $\beta$-induced fibroblast activation via JAK2/STAT3. a mRNA levels of SHP2 after overexpression in human dermal fibroblasts.

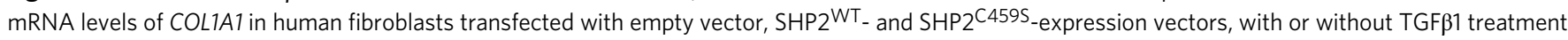
(10 $\mathrm{ng} / \mathrm{ml}$ for $24 \mathrm{~h})(n \geq 4)$. b Western blot analysis and respective quantifications for type I collagen and SHP2 in human fibroblasts transfected with empty vector, SHP2 ${ }^{W T}$ - and SHP2 ${ }^{C 459 S}$-expression vectors, with or without TGF $\beta 1$ treatment (10 ng/ml for $\left.24 \mathrm{~h}\right)$. Western blot for $\mathrm{pJAK2}$ Y1007/Y1008, pJAK2 ${ }^{Y 570}$, total JAK2, pSTAT3 ${ }^{Y 705}$ and total STAT3 with $\beta$-actin as loading control (TGF $\beta 10 \mathrm{ng} / \mathrm{ml}$ for $6 \mathrm{~h}$ ) ( $\mathrm{n}=3$ ). Results shown are representative of three independent experiments. c, d Representative images of immunofluorescence stainings for $\alpha$-SMA and stress fiber staining are shown at 400 -fold magnification (c) and quantification of $\alpha$-SMA staining intensity as well as stress fiber staining intensity (d) ( $n \geq 4)$. Horizontal scale bar, $500 \mu m$. All data are presented as median \pm s.e.m. The $p$ values are expressed as follows: $0.05>p>0.01^{\star} ; 0.01>p>0.001^{\star \star} ; p<0.001^{\star \star \star}$. Significance was determined by Mann-Whitney test. Vector: empty vector, SHP2 ${ }^{W T}$ : plasmid carrying full length of SHP2 wild-type gene, SHP2 4595 : plasmid carrying a phosphatase-dead mutant of SHP2, unstim.: unstimulated, int.: intensity 
a

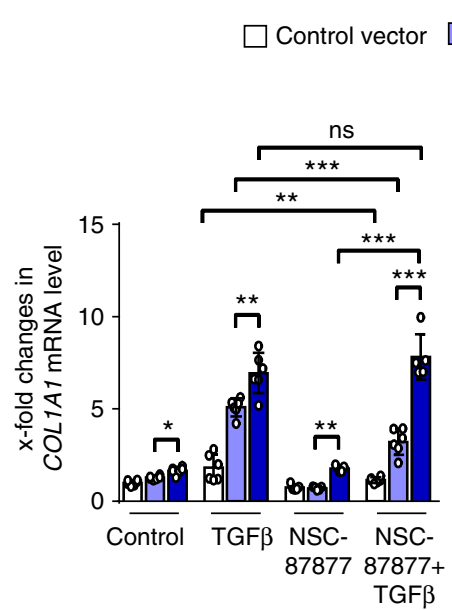

C

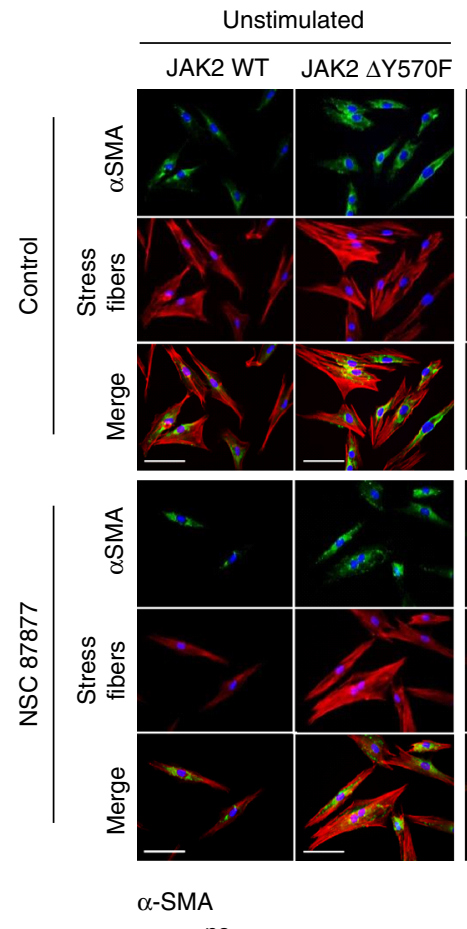

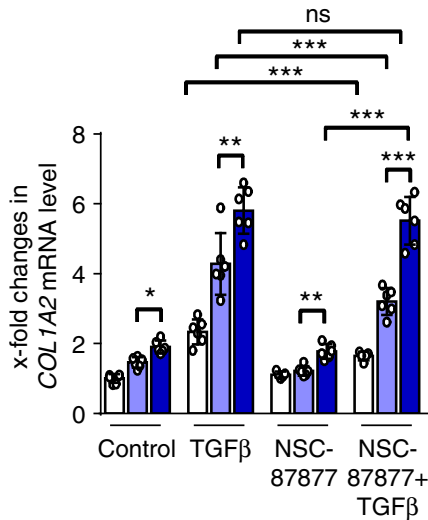

b $\square$ Control vector $\square$ JAK2 ${ }^{\mathrm{WT}}$

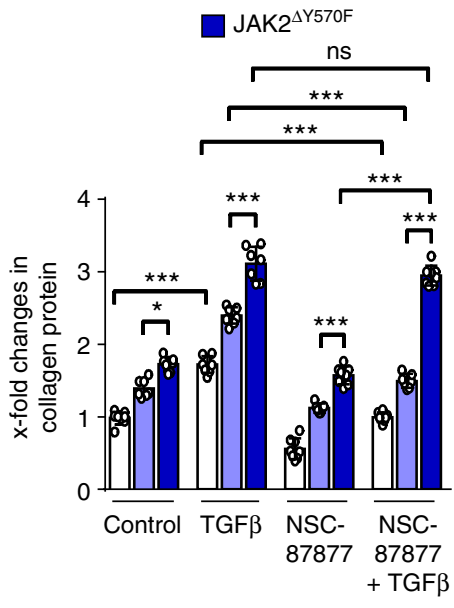

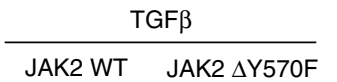
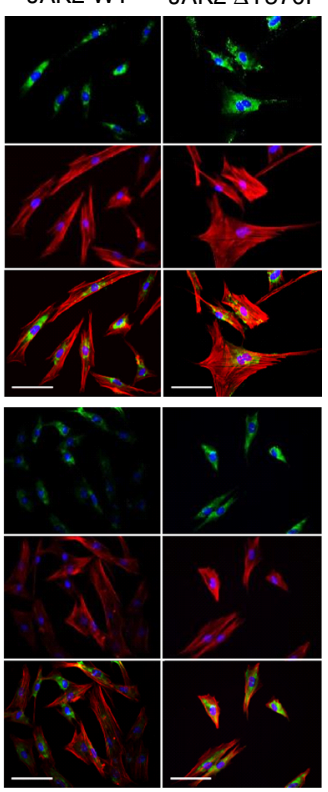

Stress fibers

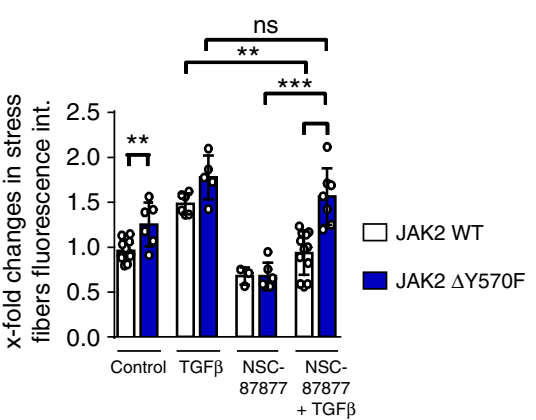

d

$\square$ Control vector $\square$ JAK2 ${ }^{\mathrm{WT}}$

JAK2 $2^{\triangle Y 570 F}$

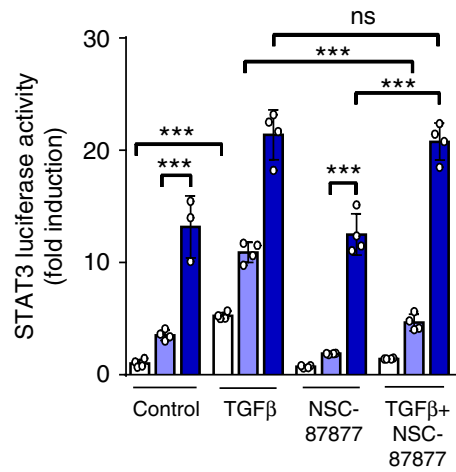

e

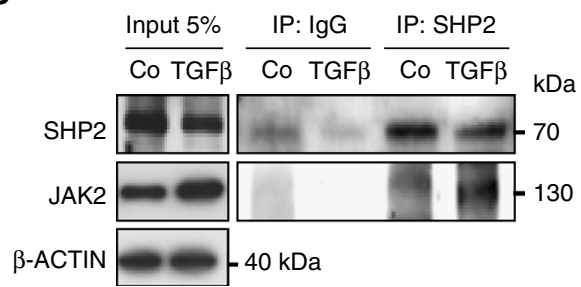

$\square$ Control $\square$ TGF $\beta$
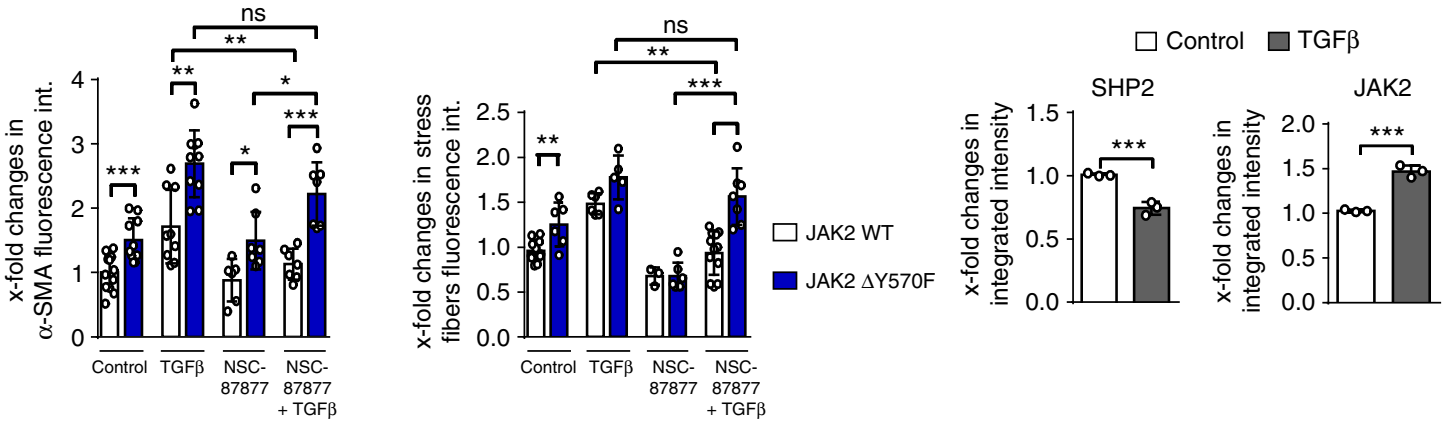

Fig. 7 Overexpression of JAK2 $2^{\triangle Y 570 F}$ prevents the inhibitory effects of SHP2 inhibitors on TGF $\beta$-induced fibroblast activation. a mRNA levels of COL1A1 and COLTA2 (TGF $10 \mathrm{ng} / \mathrm{ml}$ for $24 \mathrm{~h}$ ) ( $n \geq 5$ ). b Release of collagen protein (TGF $\beta 10 \mathrm{ng} / \mathrm{ml}$ for $24 \mathrm{~h}$ ) ( $n \geq 6$ ). c Representative images of immunofluorescence stainings for $\alpha$-SMA and stress fiber at 400-fold magnification and respective quantifications (TGF $10 \mathrm{ng} / \mathrm{ml}$ for $24 \mathrm{~h}$ ) ( $n \geq 6$ ). Horizontal scale bar, $500 \mu \mathrm{m}$. d STAT3 reporter Assay upon JAK2 WT and Y570F mutant overexpression. Cells were treated with TGF $\beta$ (10 ng/ml for $6 \mathrm{~h}$ ) and NSC-87877 $(100 \mu \mathrm{M})(n \geq 4)$. e Co-immunoprecipitation and respective quantifications of endogenous JAK2 with endogenous SHP2 in human fibroblasts stimulated with TGF $\beta(10 \mathrm{ng} / \mathrm{ml}$ for 30') $(n=3)$. Results shown are representative of $\geq 3$ independent experiments. All data are presented as median \pm s.e.m. The $p$ values are expressed as follows: $0.05>p>0.01^{\star} ; 0.01>p>0.001^{\star \star} ; p<0.001^{\star \star \star}$; ns: not significant. Significance was determined by Mann-Whitney test. JAK2WT: JAK2 Wild type, JAK2 ${ }^{\triangle Y} 570 F$ : JAK2 mutant resistant to phosphorylation at Y570, NSC-87877: SHP1/SHP2 inhibitor, Co: control unstimulated, int.: intensity, IP: immunprecipitation 
a

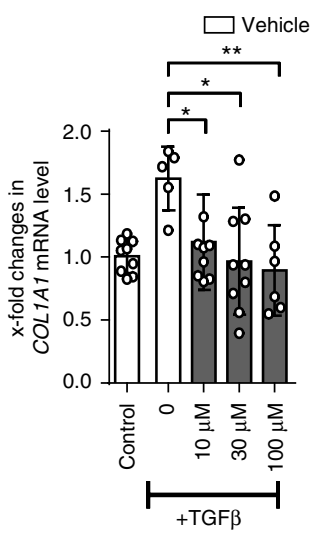

$\square$ NSC-87877

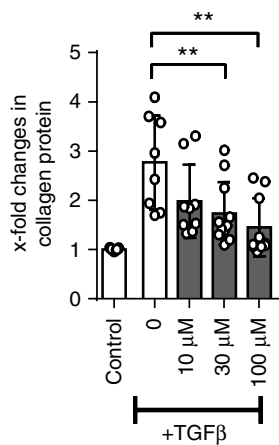

b

C
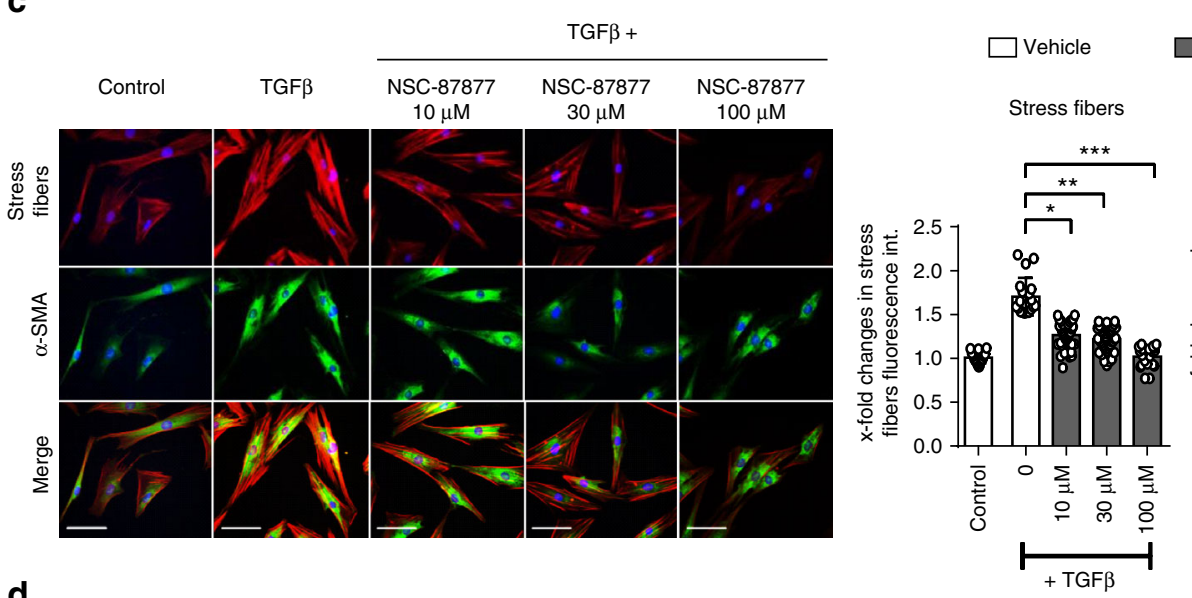

NSC-87877

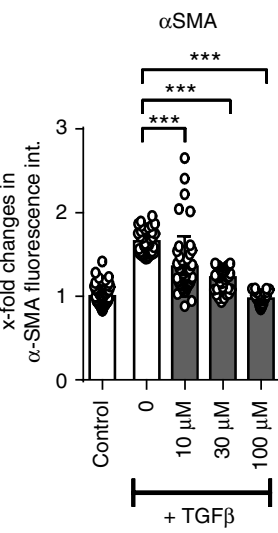

d
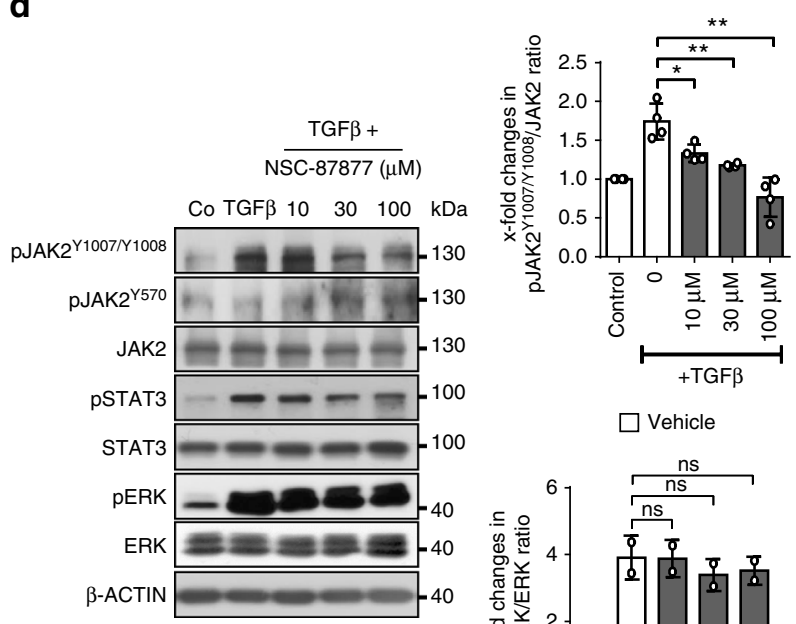

$\square$ Vehicle

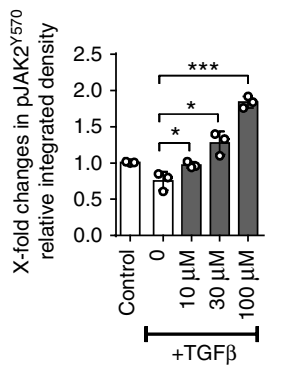

NSC-8777
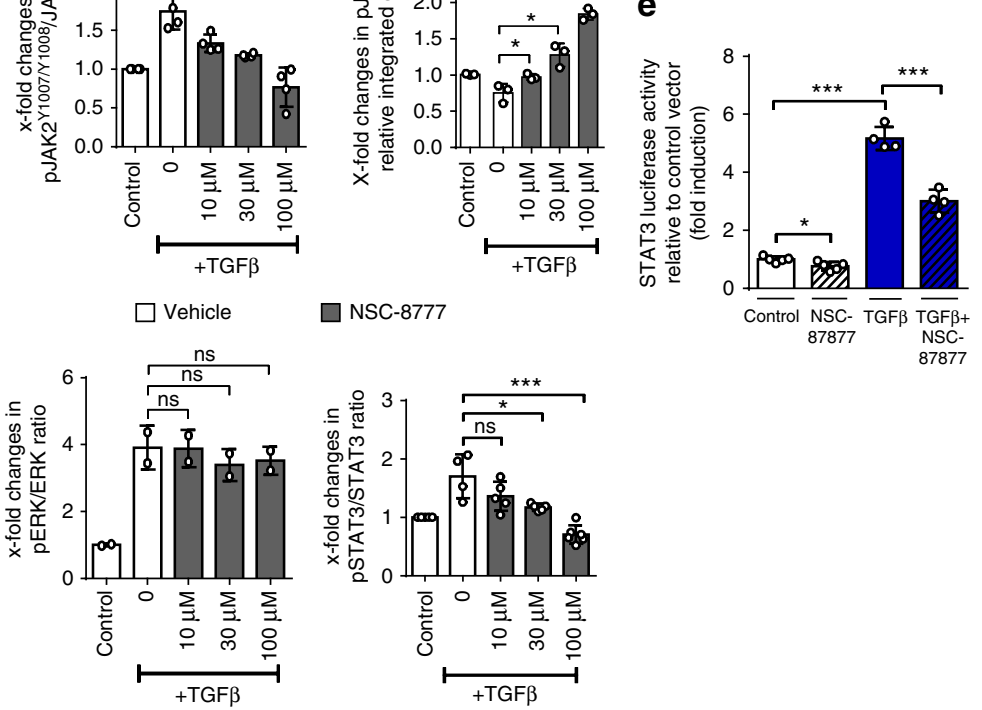

Fig. 8 Inhibition of SHP2 limits JAK2/STAT3 signaling and fibroblast activation. a Changes in the mRNA levels of COL1A1 and of collagen protein in human fibroblasts incubated with increasing doses of NSC-87877 $(10 \mu \mathrm{M}, 30 \mu \mathrm{M}$ and $100 \mu \mathrm{M})$. Fibroblasts were treated with TGF $\beta$ (10 ng/ml) for $24 \mathrm{~h}$. b ACTA2 mRNA. $(n \geq 4)$ c Representative images of immunofluorescence stainings for $\alpha$-SMA and stress fiber staining are shown at 400 -fold magnification and quantification of $\alpha$-SMA staining intensity as well as stress fiber staining intensity ( $n \geq 15)$ (TGF $\beta 10 \mathrm{ng} / \mathrm{ml}$ for $24 \mathrm{~h}$ ). Horizontal scale bar, $500 \mu \mathrm{m}$. d Representative western blots for pJAK2 ${ }^{\mathrm{Y} 1007 / \mathrm{Y} 1008}$, pJAK2 ${ }^{\mathrm{Y} 70}$, total JAK2, pSTAT3 Y705 and total STAT3 with $\beta$-actin as loading control and quantification of the results (TGF $\beta 10 \mathrm{ng} / \mathrm{ml}$ for $6 \mathrm{~h}$ ) $(n \geq 2)$. e Changes in STAT3 reporter activity ( $n \geq 6$ ) (TGF $\beta 10 \mathrm{ng} / \mathrm{ml}$ for $6 \mathrm{~h}$ ). Results shown are representative of three independent experiments All data are presented as median \pm s.e.m. The $p$ values are expressed as follows: $0.05>p>0.01^{*} ; 0.01>p$ $>0.001^{\star \star} ; p<0.001^{\star \star *}$; ns: not significant. Significance was determined by Mann-Whitney test. NSC-87877: SHP1/SHP2 inhibitor, Co: control unstimulated, int.: intensity 
a
$\mathrm{NaCl}$

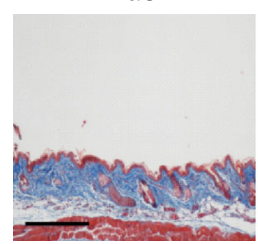

Bleo

+ NSC-87877
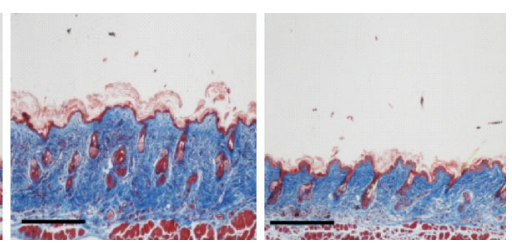

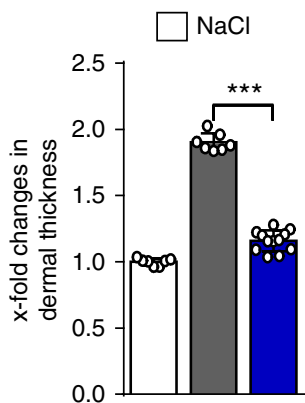

Bleo

Bleo + NSC-87877

b

AdLacZ
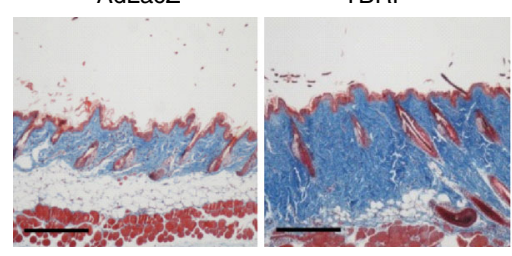

C

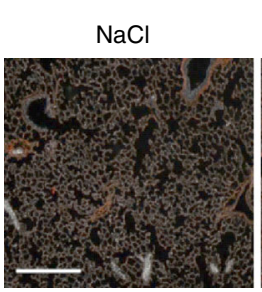

Bleo

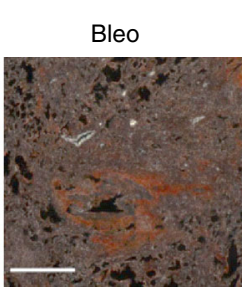

$\mathrm{TBRI}^{\mathrm{CA}}$

+ NSC-87877
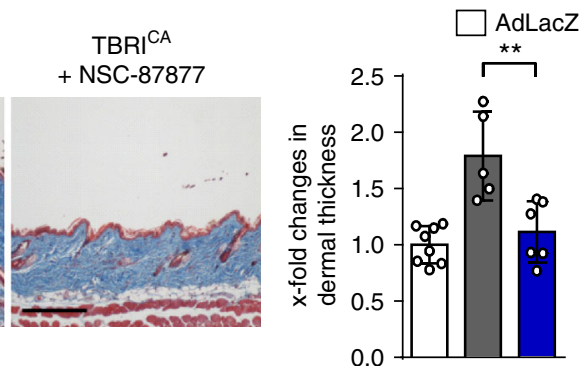
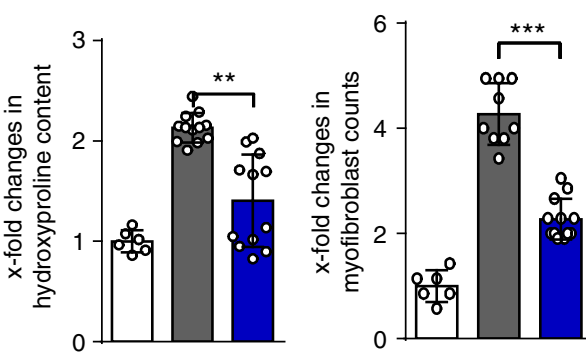

$\square$ TBRI $^{\mathrm{CA}} \square \mathrm{TBRI}^{\mathrm{CA}}+\mathrm{NSC}-87877$

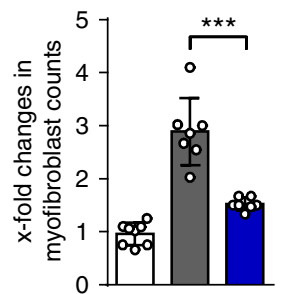

Fig. 9 Treatment with NSC-87877 ameliorates experimental fibrosis. The SHP1/SHP2 inhibitor NSC-87877 was applied at doses of $5 \mathrm{mg} / \mathrm{kg}$ q.d. a Bleomycin-induced skin ( $50 \mu \mathrm{g}$ every other day) fibrosis: representative images of Masson trichrome-stained skin shown at 100-fold magnification. Dermal thickness, hydroxyproline content and myofibroblast counts. b TBRI ${ }^{\mathrm{CA}}$-induced $\left(6.67 \times 10^{7}\right.$ IFUs every 2 weeks $)$ skin fibrosis: representative images of Masson trichrome-stained skin shown at 100 -fold magnification. Dermal thickness, hydroxyproline content and myofibroblast counts. c Bleomycin-induced lung fibrosis ( $50 \mu \mathrm{g}$ single doses): representative images of Sirius red-stained lung shown at 100 -fold magnification. Quantification of Sirius red-positive area (fibrotic area), hydroxyproline content and myofibroblast counts. All groups in all models consisted of $\geq 5$ mice each. Horizontal scale bar in all images, $500 \mu \mathrm{m}$. All data are presented as median \pm s.e.m. The $p$ values are expressed as follows: $0.05>p>0.01^{\star} ; 0.01>p>0.001^{\star \star} ; p<0.001^{\star \star \star}$. Significance was determined by Mann-Whitney test; Bleo: bleomycin, TBRICA: constitutively active TGF $\beta$ receptor type I, AdLacZ: adenovirus LacZ

patients and controls signed a consent form approved by the local institutional review board. All human studies were performed in compliance with the relevant ethical regulations

Animal studies. Mice carrying two conditional alleles of $\operatorname{Sh} 22\left(\operatorname{Sh} 2^{2 l / f f}\right)$ were crossbred with Colla2-CreER mice ${ }^{8}$. Cre-mediated recombination was induced by repeated intraperitoneal injections of $2 \mathrm{mg}$ tamoxifen over 5 days. Control groups were injected with corn oil. The role of Shp2 signaling in fibrosis was investigated in three different mouse models. (i) In the model of bleomycin-induced dermal fibrosis ( 10 weeks of age, mixed genders), fibrosis was induced by local injections of bleomycin $\left(50 \mu \mathrm{g}\right.$ every other day) for 4 weeks ${ }^{58}$. Subcutaneous injections of $0.9 \%$ $\mathrm{NaCl}$ served as control. (ii) In the TBRI ${ }^{\mathrm{CA}}$ model, injections of replication-deficient type 5 adenoviruses encoding for a constitutively active TBRI construct ${ }^{8}$ induced localized skin fibrosis (12 weeks of age, mixed genders). Mice injected with type 5 adenoviruses encoding for $L a c Z$ served as controls. $6.67 \times 10^{7}$ infectious units (IFUs) were injected intracutaneously and analyzed after 8 weeks ${ }^{59}$. (iii) TSK1 mice are a genetic model of skin fibrosis (10 weeks of age, mixed genders) with progressive accumulation of extracellular matrix in the hypodermal layer of the skin ${ }^{47}$. TSK1 mice were analyzed at an age of 10 weeks. The effect of SHP2 inhibitors NSC-87877 (Santa Cruz Technologies), PHPS1 (Calbiochem, Darmstadt, Germany), SHP099 (Chemietek, Indianapolis, USA) and 11-al (Professor Zhang, Indiana, USA) on experimental fibrosis was evaluated in three animal models: (i) In the bleomycin-induced dermal fibrosis model, treatment with intraperitoneal injection of $5 \mathrm{mg} / \mathrm{kg}$ q.d. NSC-87877 was initiated simultaneously with the first bleomycin injection and the outcome was analyzed after 3 weeks. (ii) In the $\mathrm{TBRI}^{\mathrm{CA}}$ virus-induced dermal fibrosis model (C57BL/6 background 12 weeks of age, mixed genders), treatment with intraperitoneal injections of $5 \mathrm{mg} / \mathrm{kg} /$ day NSC-
$87877,7.5 \mathrm{mg} / \mathrm{kg}$ q.d. 11 -al, $5 \mathrm{mg} / \mathrm{kg}$ q.d. PHPS1 or $75 \mathrm{mg} / \mathrm{kg}$ q.d. SHP099 via oral gavage was started simultaneously with the first virus injection and the outcome was analyzed after 8 weeks. (iii) Bleomycin-induced pulmonary fibrosis was induced by a single intra-tracheal application of bleomycin $(50 \mu \mathrm{g})$ in C57BL/6 mice (14 weeks of age, mixed genders) using a high pressure syringe (Penn-Century, Wyndmoor, PA, USA $)^{60}$. Instillation of equal volumes of $0.9 \% \mathrm{NaCl}$ served as control. Treatment with Shp2 inhibitors was started simultaneously with the instillation of bleomycin and analysis was performed after 4 weeks. In all mouse models, vehicle-treated mice served as controls. TGF- $\beta$-RI kinase inhibitor SD208 was injected intraperitoneal in a dose of $60 \mathrm{mg} / \mathrm{kg}$ q.d. All animal experiments were approved by the governments of Mittelfranken or Unterfranken, Germany. All animal experiments were performed in compliance with the relevant ethical regulations.

Cell culture. Human dermal fibroblasts were isolated from $10 \mathrm{SSc}$ patients and 10 age- and sex-matched healthy volunteers. Mouse fibroblasts were isolated from skin biopsies of Shp2-deficient (Shp $2^{f l f l}$ Colla2-CreER) mice and wild-type littermates. After enzymatic digestion of the skin biopsies with dispase II (Merck KGaA, Darmstadt, Germany), cells were cultured in Dulbecco's modified Eagle's medium/ F-12 medium containing 10\% heat-inactivated fetal calf serum, $25 \mathrm{mM}$ HEPES, $100 \mathrm{U} / \mathrm{ml}$ penicillin, $100 \mu \mathrm{g} / \mathrm{ml}$ streptomycin, $2 \mathrm{mM}$ L-glutamine, $2.5 \mu \mathrm{g} / \mathrm{ml}$ amphoteric-in B (all Gibco-Life Technologies, Darmstadt, Germany) and $0.2 \mathrm{mM}$ ascorbic acid (Sigma-Aldrich, Steinheim, Germany). Fibroblasts from passages 4-8 were used for all experiments. Cell lines were tested for mycoplasma contamination $^{61}$. Gene silencing was achieved by transfection of $3 \mu \mathrm{g}$ short interfering RNA (siRNA) duplexes using the 4D-Nucleofector (Lonza, Cologne, Germany). The siRNA duplexes: human SHP2 5'-GGU ACA UCG ACU UCC UCU A-3' 
a

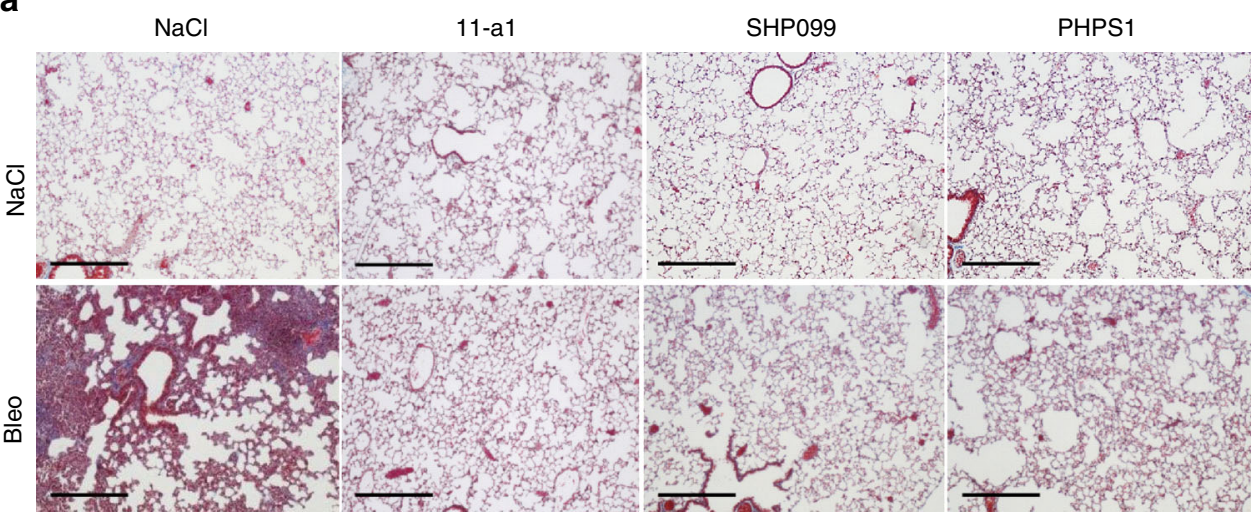

$\square \mathrm{NaCl} \square$ Bleo
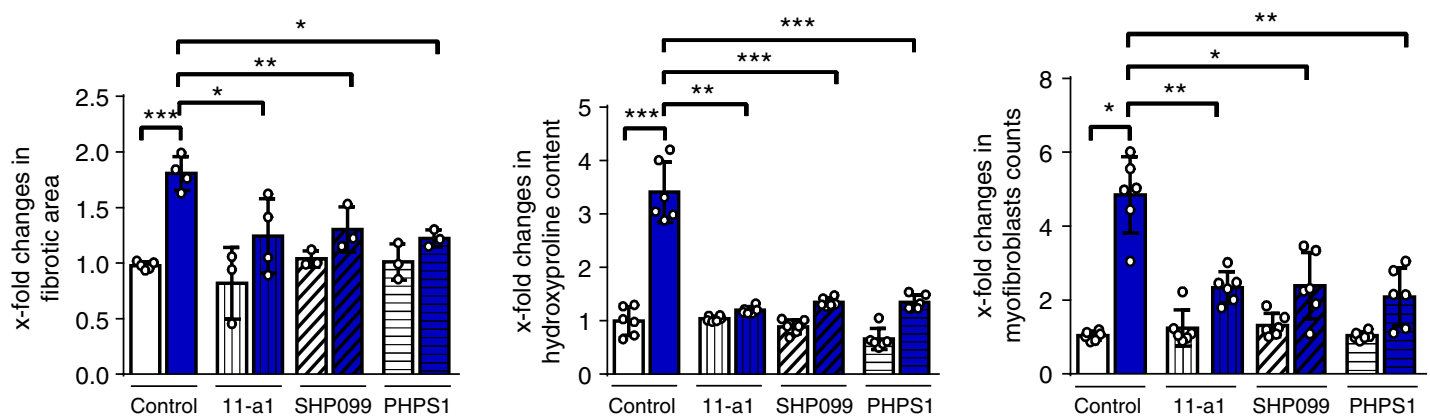

b
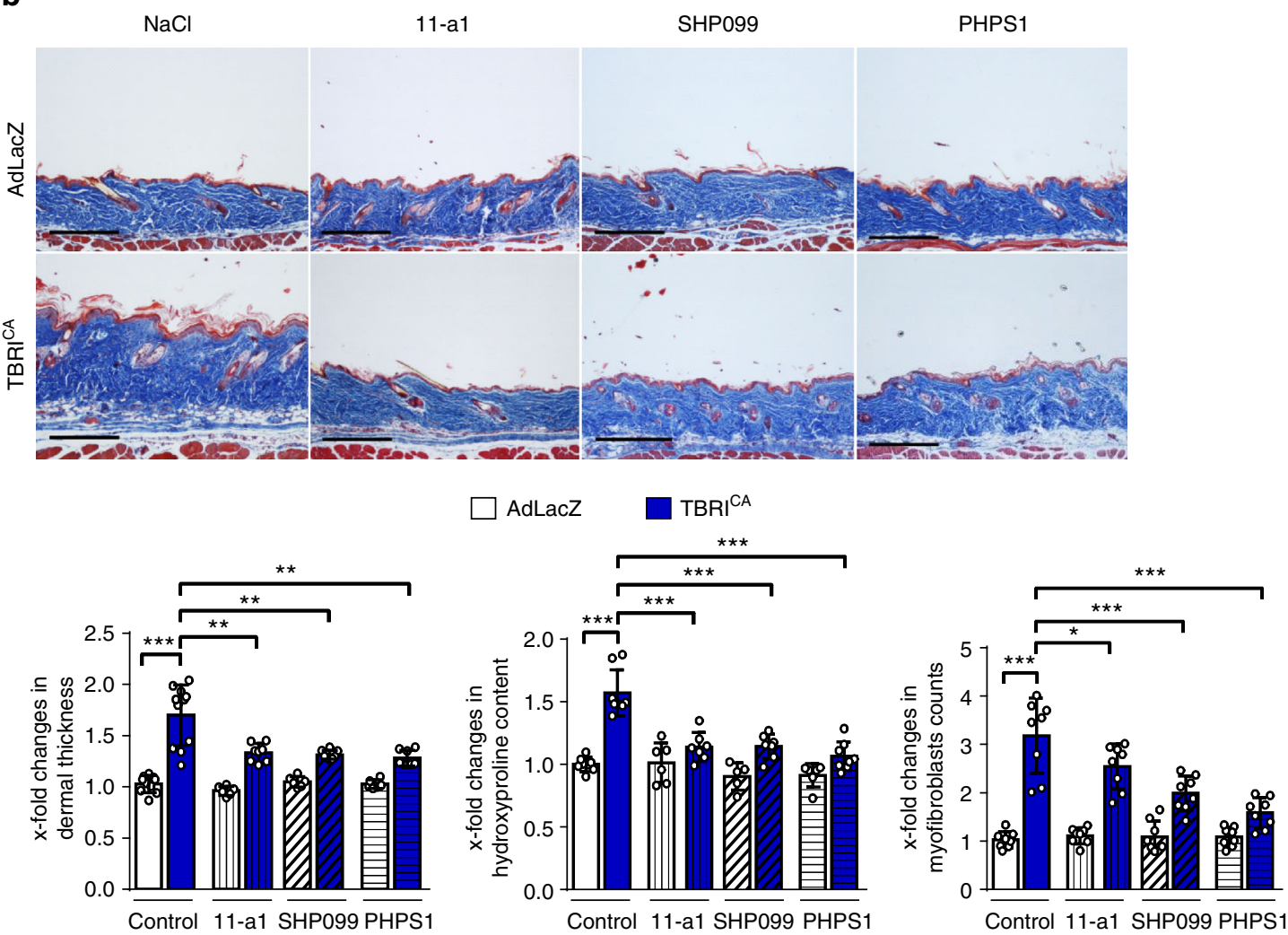

(forward), 5'-UAG AGG AAG UCG AUG UAC C-3' (reverse); non-targeting siRNAs (Life Technologies, Darmstadt, Germany) served as controls. Cre-mediated recombination in murine fibroblasts isolated from $S h p 2^{f l / f l}$ mice was induced by infection with type 5 adenoviral vectors encoding for Cre recombinase (AdCre - 80 IFUs/fibroblast). Type 5 adenoviral vectors encoding for LacZ (AdLacZ) served as controls. In selective experiments, cells were incubated with recombinant TGF $\beta$ (10 ng/ml) (PeproTech, Hamburg, Germany).

SHP2 activity was inhibited in fibroblasts using the following inhibitors: NSC$87877(100 \mu \mathrm{M})$, SHP099 $(1.4 \mu \mathrm{M})$ and 11-a1 $(0.2 \mu \mathrm{M})$. Cells were pretreated with inhibitors $2 \mathrm{~h}$ before addition of TGF $\beta$. JAK2 was inhibited using the JAK2 inhibitor TG101209 (500 nM) (Selleckchem, Houston, USA).

Quantitative real time-PCR. Gene expression was quantified by SYBR Green real time-PCR using the MX3005P Detection System (Agilent Technologies, Böblingen, Germany). Primers are listed in the Supplementary table 1. Samples without enzyme in the reverse transcription reaction (Non-RT controls) were used as negative controls. Unspecific signals caused by primer dimers were excluded by 
Fig. 10 Selective inhibition of Shp2 ameliorates experimental fibrosis. The following doses of SHP2 inhibitors were applied: PHPS1 (5 mg/kg q.d.), SHP099 ( $75 \mathrm{mg} / \mathrm{kg}$ q.d.) and 11-a1 (7.5 mg/kg q.d.). a Bleomycin-induced pulmonary fibrosis (50 $\mu$ g single doses): representative images of Masson trichromestained skin shown at 100-fold magnification; fibrotic area, hydroxyproline content and myofibroblast counts. b TBRI CA $_{\text {-induced dermal fibrosis }}\left(6.67 \times 10^{7}\right.$ IFUs every 2 weeks): representative images of Masson trichrome-stained skin shown at 100-fold magnification; Dermal thickness, myofibroblast counts and hydroxyproline content. All groups in both models consisted of $\geq 4$ mice each. Horizontal scale bar for all images, $500 \mu \mathrm{m}$ All data are presented as median \pm s.e.m. The $p$ values are expressed as follows: $0.05>p>0.01^{\star} ; 0.01>p>0.001^{\star \star} ; p<0.001^{\star \star \star}$. Significance was determined by Mann-Whitney test. Bleo: bleomycin, AdLacZ: adenovirus LacZ, TBRICA: constitutively active TGF $\beta$ receptor type I

non-template controls and by dissociation curve analysis ${ }^{62} \cdot \beta$-Actin was used to normalize for the amounts of complementary DNA within each sample.

Western blot analysis. The protein concentration of cell lysates was determined by amido black assays or Bradford protein assay (\#5000001 BIO-RAD, Hercules, USA). Proteins were separated by sodium dodecyl sulfate-polyacrylamide gel electrophoresis (SDS-PAGE) and transferred to a polyvinylidene difluoride (PVDF) membrane. The membrane was incubated with antibodies against SHP2 (1:1000), pJAK2 ${ }^{Y 1007 / Y 1008}$ (1:500), JAK2 (1:1000) (\# sc-7384, \#sc-280, \#sc-16566R, \#sc-278, Santa Cruz Technologies, Heidelberg, Germany), pJAK2 (Y570) (1:500) (\#CPA1629, Cohesion Biosciences), Collagen I (1:1000) (\#ab138492, Abcam Cambridge, UK), pSTAT3 (1:1000), STAT3 (1:1000) (\#9145, \#9139, Cell Signaling, Boston, USA) and horseradish peroxidase (HRP)-conjugated secondary antibodies (Dako, Hamburg, Germany). Blots were visualized by ECL. $\beta$-Actin was used as loading control. Western blots were quantified using the ImageJ Software (version 1.41). The uncropped scans of western blots presented in the figures are shown in Supplementary Fig. 5 and 6 .

Co-immunoprecipitation. After stimulation of fibroblasts with TGF $\beta(10 \mathrm{ng} / \mathrm{ml})$ for $3 \mathrm{~min}$, cells were collected in lysis buffer (Tris- $\mathrm{HCl} 50 \mathrm{mM}, \mathrm{NaCl} 150 \mathrm{mM}$, EDTA $1 \mathrm{mM}$, NP-40 1\%, dithiothreitol (DTT) $1 \mathrm{mM}$ and phenylmethylsulfonyl fluoride $1 \mathrm{mM}$ ). Five percent of the lysates were used as input. Then, $500 \mu \mathrm{g}$ of protein lysate (Cell extract) was first incubated with $2 \mu \mathrm{g}$ of antibodies against either SHP2 or serum IgG (all from Santa Cruz Biotechnology, Heidelberg, Germany) for $2 \mathrm{~h}$ at $4{ }^{\circ} \mathrm{C}$ under rotation. Subsequently, $30 \mu \mathrm{l}$ of Protein A/G Sepharose was added to the samples. Unbound proteins were removed by washing with Tris buffer (Tris-HCl 50 mM, NaCl 150 mM, EDTA 1 mM, NP-40 1\%). Sepharose-bound protein complexes were separated via SDS-PAGE followed by western blotting on a PVDF membrane. Proteins were visualized via ECL prime kit (GE Healthcare, Braunschweig, Germany).

Phosphatase activity assay (PTP activity assay). Protein samples were isolated from human fibroblasts and SHP2 immunprecipitated as described above. Paranitrophenyl phosphate (p-NPP; Sigma-Aldrich) was used as enzyme substrate. The SHP2 immune complexes were washed three times in Tris buffer and once in phosphatase buffer (30 mM HEPES pH 7.4, $120 \mathrm{mM} \mathrm{NaCl}$ ). Afterwards, samples were resuspended with $200 \mu \mathrm{l}$ of phosphatase assay buffer $(30 \mathrm{mM}$ HEPES pH 7.4, $120 \mathrm{mM} \mathrm{NaCl}, 5 \mathrm{mM}$ p-NPP, $1 \mathrm{mM}$ DTT and $65 \mathrm{ng} / \mu \mathrm{l}$ bovine serum albumin) and incubated at $30{ }^{\circ} \mathrm{C}$ for 30 to $90 \mathrm{~min}$. Hydrolysis of p-NPP was determined by reading the absorbance at $405 \mathrm{~nm}$ with a microtiter plate reader (spectrophotometer) ${ }^{20}$. A Recombinant Human SHP2 (R\&D Systems, Minneapolis, USA) was used to generate a standard curve $(0,1,2,4,6,8$ and $10 \mathrm{ng})$.

Quantification of collagen protein. The amount of soluble collagen in cell culture supernatants was quantified using the SirCol collagen assay (Biocolor, Belfast, Northern Ireland). The total collagen content of tissue samples was determined by hydroxyproline assays using the chloramines-T method ${ }^{28,63}$. In brief, samples were digested with $6 \mathrm{M} \mathrm{HCl}$ for $4-6 \mathrm{~h}$. Samples were centrifuged to remove debris and $\mathrm{pH}$ of the solution is adjusted to 7 . Samples were hydrolyzed by incubation at $60^{\circ} \mathrm{C}$ for $30 \mathrm{~min}$. The cloramines- $\mathrm{T}$ was added to the hydrolyzate to allow oxidation followed by the addition of Ehrlich's aldehyde reagent. The absorbance intensity of each sample was analyzed at $550 \mathrm{~nm}$ using a microtiter plate reader spectrophotometer.

Immunohistochemistry and immunofluorescence staining. Formalin-fixed, paraffin-embedded skin sections or fibroblasts fixed in 4\% paraformaldehyde and permeabilized by $0.25 \%$ Triton X-100 were stained with antibodies against $\alpha$-SMA (1:1000) (Life Technologies), SHP2 (1:200) (\#sc-280, Santa Cruz Technologies, Heidelberg, Germany), P4H $\beta$ (1:200) (\#AP08767PU-N, Acris Antibodies, Herford, Germany), vimentin (1:500) (\#20346, Abcam), CD31 (1:200) (\#AF3628, R\&D Systems, Minneapolis, USA) and CD45 (1:200) (\#MA5-17687, Thermo Fisher, Massachusetts, USA). HRP-conjugated or Alexa Fluor antibodies (1:200) (Life Technologies) were used as secondary antibodies. Irrelevant isotype-matched antibodies served as controls. Stress fibers were visualized with rhodamineconjugated phalloidin (\#R415, Sigma-Aldrich). Nuclei were counterstained using 4',6-diamidino-2-phenylindole (DAPI; Santa Cruz Biotechnology). The staining was analyzed using a Nikon Eclipse $80 \mathrm{i}$ microscope (Nikon, Badhoevedorp, Netherlands). Voronoi tessellation of in vivo immunofluorescence pictures were performed using the ImageJ2 software $\mathrm{e}^{64,65}$.

Plasmid and reporter constructs. $\mathrm{pJ} 3-\mathrm{SHP} 2{ }^{\mathrm{C} 459 \mathrm{~S}}$ and $\mathrm{pJ} 3-\mathrm{SHP} 2 \mathrm{WT}$ were provided by Ben Neel ${ }^{66}$ via Addgene (Cambridge, USA, plasmids \#8319 and \#8317, respectively). The Luciferase reporter plasmids p-TA-luc and pSTAT3-TA-luc were purchased from ClonTech (Mountain View, CA, USA). Fibroblasts were transfected with $5 \mu \mathrm{g}$ of either plasmid or empty control vectors using the Amaxa $4 \mathrm{D}$ Nucleofector (Amaxa, Cologne, Germany). The transfection efficiency was determined by co-transfection with vectors encoding for $\beta$-galactosidase (Promega, Mannheim, Germany).

The plasmid pCMV3-Flag-JAK2 encoding the human JAK2 was purchased from Sino Biological (Beijing, China). In vitro mutagenesis of JAK2 was performed using the QuickChange Multi site-directed mutagenesis kit (Agilent Technologies) to yield JAK2 $\triangle \mathrm{Y} 570 \mathrm{~F}$, a JAK2 mutant that cannot be phosphorylated at the inhibitory site Y570. After verification of the correct sequence, the construct was transfected in dermal human fibroblasts using Amaxa 4D-Nucleofector for overexpression studies.

Histological analyses. Skin sections were stained with hematoxylin/eosin or trichrome. The dermal thickness was analyzed at four different sites in each mouse in a blinded manner ${ }^{67}$. Dermal thickness was analyzed with a Nikon Eclipse $80 \mathrm{i}$ microscope (Nikon) at 100-fold magnification by measuring the distance between the epidermal-dermal junction and the dermal-subcutaneous fat junction at sites of induration at three consecutive skin sections of each animal ${ }^{68}$. For direct visualization of collagen fibers, Sirius Red staining was performed (Sigma-Aldrich)

Statistics. All data are presented as median \pm s.e.m, and differences between the groups were tested for their statistical significance by Mann-Whitney $U$-test. The $p$ values of less than 0.05 were considered as statistically significant; $p$ values are expressed as follows: $0.05>p>0.01^{*} ; 0.01>p>0.001^{* *} ; p<0.001^{* * *}$. GraphPad Prism software 7.0 was used for statistical analysis. The sample size was estimated based on previous experiments. No statistical method was used to predetermine sample size.

Experiments were not done in a blinded fashion except when specifically indicated. There were no exclusion criteria for the human and animal experiments Mice were stratified according to sex and then randomized into the different treatment groups. Cells from human donors were also randomized.

Data availability. The datasets generated and analyzed during the current study are available from the corresponding authors on reasonable request.

Received: 30 October 2017 Accepted: 25 July 2018

Published online: 14 August 2018

\section{References}

1. Distler, J. H. et al. Review: frontiers of antifibrotic therapy in systemic sclerosis. Arthritis Rheumatol. (Hoboken, NJ) 69, 257-267 (2017).

2. Wynn, T. A. Cellular and molecular mechanisms of fibrosis. J. Pathol. 214, 199-210 (2008).

3. Gurtner, G. C., Werner, S., Barrandon, Y. \& Longaker, M. T. Wound repair and regeneration. Nature 453, 314-321 (2008).

4. Gabrielli, A., Avvedimento, E. V. \& Krieg, T. Scleroderma. N. Engl. J. Med. 360, 1989-2003 (2009).

5. Feghali, C. A. \& Wright, T. M. Identification of multiple, differentially expressed messenger RNAs in dermal fibroblasts from patients with systemic sclerosis. Arthritis Rheum. 42, 1451-1457 (1999).

6. Mori, Y., Chen, S. J. \& Varga, J. Expression and regulation of intracellular SMAD signaling in scleroderma skin fibroblasts. Arthritis Rheum. 48, 1964-1978 (2003)

7. Leask, A. \& Abraham, D. J. TGF-beta signaling and the fibrotic response. FASEB J. 18, 816-827 (2004). 
8. Sonnylal, S. et al. Postnatal induction of transforming growth factor beta signaling in fibroblasts of mice recapitulates clinical, histologic, and biochemical features of scleroderma. Arthritis Rheum. 56, 334-344 (2007).

9. Tartaglia, M. et al. Mutations in PTPN11, encoding the protein tyrosine phosphatase SHP-2, cause Noonan syndrome. Nat. Genet. 29, 465-468 (2001).

10. Hof, P., Pluskey, S., Dhe-Paganon, S., Eck, M. J. \& Shoelson, S. E. Crystal structure of the tyrosine phosphatase SHP-2. Cell 92, 441-450 (1998).

11. Grossmann, K. S., Rosario, M., Birchmeier, C. \& Birchmeier, W. The tyrosine phosphatase Shp2 in development and cancer. Adv. Cancer Res. 106, 53-89 (2010).

12. Qu, C. K. Role of the SHP-2 tyrosine phosphatase in cytokine-induced signaling and cellular response. Biochim. Biophys. Acta 1592, 297-301 (2002).

13. Kang, H. J. et al. SHP2 is induced by the HBx-NF- $\mathrm{kB}$ pathway and contributes to fibrosis during human early hepatocellular carcinoma development. Oncotarget 8, 27263-27276 (2017).

14. Tajan, M., de Rocca Serra, A., Valet, P., Edouard, T. \& Yart, A. SHP2 sails from physiology to pathology. Eur. J. Med. Genet. 58, 509-525 (2015).

15. Wen-Qing, H. et al. Structure, function, and pathogenesis of SHP2 in developmental disorders and tumorigenesis. Curr. Cancer Drug. Targets 14 567-588 (2014).

16. Ivins Zito, C., Kontaridis, M. I., Fornaro, M., Feng, G. S. \& Bennett, A. M. SHP-2 regulates the phosphatidylinositide 3'-kinase/Akt pathway and suppresses caspase 3-mediated apoptosis. J. Cell. Physiol. 199, 227-236 (2004).

17. Zhang, S. Q. et al. Receptor-specific regulation of phosphatidylinositol 3'kinase activation by the protein tyrosine phosphatase Shp2. Mol. Cell. Biol. 22, 4062-4072 (2002).

18. Stewart, R. A. et al. Phosphatase-dependent and -independent functions of Shp2 in neural crest cells underlie LEOPARD syndrome pathogenesis. Dev. Cell 18, 750-762 (2010).

19. Sarkozy, A., Digilio, M. C. \& Dallapiccola, B. Leopard syndrome. Orphanet. J. Rare. Dis. 3, 13 (2008)

20. Wang, J. et al. Inhibition of SHP2 ameliorates the pathogenesis of systemic lupus erythematosus. J. Clin. Invest. 126, 2077-2092 (2016).

21. Stanford, S. M. et al. Protein tyrosine phosphatase expression profile of rheumatoid arthritis fibroblast-like synoviocytes: a novel role of SH2 domaincontaining phosphatase 2 as a modulator of invasion and survival. Arthritis Rheum. 65, 1171-1180 (2013).

22. Maeshima, K. et al. Abnormal PTPN11 enhancer methylation promotes rheumatoid arthritis fibroblast-like synoviocyte aggressiveness and joint inflammation. JCI Insight 1, e86580 (2016).

23. Varga, J. \& Abraham, D. Systemic sclerosis: a prototypic multisystem fibrotic disorder. J. Clin. Invest. 117, 557-567 (2007)

24. Aceto, N. et al. Tyrosine phosphatase SHP2 promotes breast cancer progression and maintains tumor-initiating cells via activation of key transcription factors and a positive feedback signaling loop. Nat. Med. 18, 529-537 (2012).

25. Lehmann, U. et al. SHP2 and SOCS3 contribute to Tyr-759-dependent attenuation of interleukin-6 signaling through gp130. J. Biol. Chem. 278, 661-671 (2003).

26. Ali, S., Nouhi, Z., Chughtai, N. \& Ali, S. SHP-2 regulates SOCS-1-mediated Janus kinase-2 ubiquitination/degradation downstream of the prolactin receptor. J. Biol. Chem. 278, 52021-52031 (2003).

27. Godeny, M. D. et al. The N-terminal SH2 domain of the tyrosine phosphatase, SHP-2, is essential for Jak2-dependent signaling via the angiotensin II type AT1 receptor. Cell. Signal. 19, 600-609 (2007).

28. Zhang, Y. et al. JAK1-dependent transphosphorylation of JAK2 limits the antifibrotic effects of selective JAK2 inhibitors on long-term treatment. Ann. Rheum. Dis. 76, 1467-1475 (2017).

29. Dees, C. et al. JAK-2 as a novel mediator of the profibrotic effects of transforming growth factor beta in systemic sclerosis. Arthritis Rheum. 64, 3006-3015 (2012).

30. Chakraborty, D. et al. Activation of STAT3 integrates common profibrotic pathways to promote fibroblast activation and tissue fibrosis. Nat. Commun. 8 , 1130 (2017).

31. Zeng, L. F. et al. Therapeutic potential of targeting the oncogenic SHP2 phosphatase. J. Med. Chem. 57, 6594-6609 (2014).

32. Chen, Y. N. et al. Allosteric inhibition of SHP2 phosphatase inhibits cancers driven by receptor tyrosine kinases. Nature 535, 148-152 (2016).

33. Hellmuth, K. et al. Specific inhibitors of the protein tyrosine phosphatase Shp2 identified by high-throughput docking. Proc. Natl. Acad. Sci. USA 105, 7275-7280 (2008).

34. Tibaldi, E. et al. The tyrosine phosphatase SHP-1 inhibits proliferation of activated hepatic stellate cells by impairing PDGF receptor signaling. Biochim. Biophys. Acta 1843, 288-298 (2014).

35. Su, T. H. et al. Src-homology protein tyrosine phosphatase-1 agonist, SC-43, reduces liver fibrosis. Sci. Rep. 7, 1728 (2017).
36. Li, S. et al. SHP2 positively regulates TGFbeta1-induced epithelialmesenchymal transition modulated by its novel interacting protein Hook1. J. Biol. Chem. 289, 34152-34160 (2014).

37. Tang, J. et al. Fluorofenidone protects against renal fibrosis by inhibiting STAT3 tyrosine phosphorylation. Mol. Cell. Biochem. 407, 77-87 (2015).

38. Ma, X. Q. et al. Effects of matrine on Jak-Stat signaling transduction pathways in bleomycin-induced pulmonary fibrosis. Afr. J. Tradit. Complem 10, 442-448 (2013).

39. Feener, E. P., Rosario, F., Dunn, S. L., Stancheva, Z. \& Myers, M. G. Jr. Tyrosine phosphorylation of Jak2 in the JH2 domain inhibits cytokine signaling. Mol. Cell. Biol. 24, 4968-4978 (2004).

40. Zhang, Y. et al. Inhibition of casein kinase II reduces TGF $\beta$ induced fibroblast activation and ameliorates experimental fibrosis. Ann. Rheum. Dis. 74, 936 (2015).

41. Qin, X. J. et al. Protein tyrosine phosphatase SHP2 regulates TGF-beta1 production in airway epithelia and asthmatic airway remodeling in mice. Allergy 67, 1547-1556 (2012).

42. Sun, X. et al. Shp2 plays a critical role in IL-6-induced EMT in breast cancer cells. Int. J. Mol. Sci. 18, E395 (2017).

43. Kawano, H. et al. Myocardial fragmentation associated with disruption of the Z-band in hypertrophic cardiomyopathy in Noonan syndrome. Cardiovasc. Pathol. 25, 329-332 (2016).

44. Hudsmith, L. E. et al. Hypertrophic cardiomyopathy in Noonan Syndrome closely mimics familial hypertrophic cardiomyopathy due to sarcomeric mutations. Int. J. Cardiovasc. Imaging 22, 493-495 (2006).

45. Elgohary, M., Bradshaw, P. \& Ahmad, N. Anterior uveitis and congenital fibrosis of the extraocular muscles in a patient with Noonan syndrome. $J$. Postgrad. Med. 51, 319-321 (2005).

46. Naing, A. et al. Phase I dose escalation study of sodium stibogluconate (SSG), a protein tyrosine phosphatase inhibitor, combined with interferon alpha for patients with solid tumors. J. Cancer 2, 81-89 (2011).

47. Soare, A., Ramming, A., Avouac, J. \& Distler, J. H. Updates on animal models of systemic sclerosis. Jsrd 1, 266-276 (2016).

48. Zhao, L. et al. Shp2 deficiency impairs the inflammatory response against haemophilus influenzae by regulating macrophage polarization. J. Infect. Dis. 214, 625-633 (2016).

49. Tao, B. et al. Myeloid-specific disruption of tyrosine phosphatase Shp2 promotes alternative activation of macrophages and predisposes mice to pulmonary fibrosis. J. Immunol. 193, 2801 (2014).

50. Chia, J. J. \& Lu, T. T. Update on macrophages and innate immunity in scleroderma. Curr. Opin. Rheumatol. 27, 530-536 (2015).

51. Wynn, T. A. \& Vannella, K. M. Macrophages in tissue repair, regeneration, and fibrosis. Immunity 44, 450-462 (2016).

52. Tzouvelekis, A. et al. SH2 domain-containing phosphatase-2 is a novel antifibrotic regulator in pulmonary fibrosis. Am. J. Respir. Crit. Care Med. 195, 500-514 (2017).

53. Won, K. J. et al. Protein tyrosine phosphatase SHP-2 is positively involved in platelet-derived growth factor-signaling in vascular neointima formation via the reactive oxygen species-related pathway. J. Pharmacol. Sci. 115, 164-175 (2011).

54. Lee, Y. H., Mungunsukh, O., Tutino, R. L., Marquez, A. P. \& Day, R. M. Angiotensin-II-induced apoptosis requires regulation of nucleolin and Bcl-x (L) by SHP-2 in primary lung endothelial cells. J. Cell. Sci. 123, 1634-1643 (2010).

55. Manetti, M. et al. Inactivation of urokinase-type plasminogen activator receptor ( $\mathrm{UPAR}$ ) gene induces dermal and pulmonary fibrosis and peripheral microvasculopathy in mice: a new model of experimental scleroderma? Ann. Rheum. Dis. 73, 1700-1709 (2013).

56. Maurer, B., Distler, J. H. \& Distler, O. The Fra-2 transgenic mouse model of systemic sclerosis. Vasc. Pharmacol. 58, 194-201 (2013).

57. van den Hoogen, F. et al. 2013 Classification criteria for systemic sclerosis: an American college of rheumatology/European league against rheumatism collaborative initiative. Ann. Rheum. Dis. 72, 1747-1755 (2013).

58. Yamamoto, T. et al. Animal model of sclerotic skin. I: Local injections of bleomycin induce sclerotic skin mimicking scleroderma. J. Invest. Dermatol. 112, 456-462 (1999).

59. Palumbo-Zerr, K. et al. Orphan nuclear receptor NR4A1 regulates transforming growth factor-beta signaling and fibrosis. Nat. Med. 21, 150-158 (2015).

60. Liang, R. et al. The transcription factor GLI2 as a downstream mediator of transforming growth factor-beta-induced fibroblast activation in SSc. Ann. Rheum. Dis. 76, 756-764 (2017)

61. Dees $\mathrm{C}$ et al. Platelet-derived serotonin links vascular disease and tissue fibrosis. J. Exp. Med. 208, 961-972 (2011).

62. Akhmetshina, A. et al. Activation of canonical Wnt signalling is required for TGF-beta-mediated fibrosis. Nat. Commun. 3, 735 (2012).

63. Zerr, P. et al. Inhibition of hedgehog signaling for the treatment of murine sclerodermatous chronic graft-versus-host disease. Blood 120, 2909-2917 (2012). 
64. Faisal, T. R., Hristozov, N., Rey, A. D., Western, T. L. \& Pasini, D. Experimental determination of Philodendron melinonii and Arabidopsis thaliana tissue microstructure and geometric modeling via finite-edge centroidal Voronoi tessellation. Phys. Rev. E 86, 031921 (2012).

65. $\mathrm{Wu}, \mathrm{M}$. et al. Single- and two-phase flow in microfluidic porous media analogs based on Voronoi tessellation. Lab Chip 12, 253-261 (2012).

66. Bennett, A. M., Hausdorff, S. F., O’Reilly, A. M., Freeman, R. M. \& Neel, B. G. Multiple requirements for SHPTP2 in epidermal growth factor-mediated cell cycle progression. Mol. Cell. Biol. 16, 1189-1202 (1996).

67. Dees, C. et al. The Wnt antagonists DKK1 and SFRP1 are downregulated by promoter hypermethylation in systemic sclerosis. Ann. Rheum. Dis. 73, 1232-1239 (2014).

68. Zerr, P. et al. Vitamin D receptor regulates TGF-beta signalling in systemic sclerosis. Ann. Rheum. Dis. 74, e20 (2015).

\section{Acknowledgements}

We thank Rita Weinkam, Regina Kleinlein, Katja Dreißigacker and Rossella Mancuso for excellent technical assistance. This work was supported by grants DI 1537/5-1, DI 1537/ 7-1, DI 1537/8-1, DI 1537/9-1, DI 1537/11-1, DE 2414/2-1, RA 2506/3-1 and AK 144/ 2-1 of the German Research Foundation, CRC1181 (project C01) of the German Research Foundation, grant NIH RO1 CA207288 (to Z.-Y.Z) of the National Institutes of Health (USA), grants A57, J40 and A64 of the IZKF in Erlangen, grant 2013.056.1 of the Wilhelm-Sander-Foundation, grants 2014_A47, 2014_A248 and 2014_A184 of the ElseKröner-Fresenius-Foundation, grant 14-12-17-1-Bergmann of the ELAN-Foundation Erlangen and a Career Support Award of Medicine of the Ernst Jung Foundation.

\section{Author contributions}

A.Z., J.H. and J.H.W.D. designed the research; A.Z., J.H., A.-H.G., A.-E.M., T.T.-M., X.X., Y.-N.L., Z.-Y.Z., J.L., C.-W.C., C.B., C.D., A.R., C.B., W.B. and K.G. performed the research; J.H., A.Z., C.B., A.-H.G., C.D., O.D., G.S. and J.H.W.D. analyzed the data; A.Z., J.H. and J.H.W.D. wrote the manuscript.

\section{Additional information} Supplementary Information accompanies this paper at https://doi.org/10.1038/s41467018-05768-3
Competing interests: None of the authors has any direct financial interest related to SHP2. O.D. has consultancy relationships and/or has received research funding for the treatment of SSc from Actelion, Pfizer, Ergonex, BMS, Sanofi-Aventis, United BioSource Corporation, Roche/Genentech, Medac, Biovitrium, Boehringer Ingelheim, Novartis, 4D Science, Active Biotech, Bayer, Sinoxa, Serodapharm, EpiPharm, GSK, Pharmacyclics and Biogen. J.H.W.D. has consultancy relationships for fibrotic diseases with Actelion, Active Biotech, Anamar, Bayer Pharma, Boehringer Ingelheim, Celgene, Galapagos, Inventiva, JB Therapeutics, Medac, Pfizer, RuiYi and UCB in the area of potential treatments of SSc. J.H.W.D. has received research funding for projects on fibrosis from Anamar, Active Biotech, Array Biopharma, BMS, Bayer Pharma, Boehringer Ingelheim, Celgene, GSK, Novartis, Sanofi-Aventis, UCB in the area of potential treatments of SSc. J.H.W.D. is stock owner of $4 \mathrm{D}$ Science $\mathrm{GmbH}$. The remaining authors declare no competing interests.

Reprints and permission information is available online at http://npg.nature.com/ reprintsandpermissions/

Publisher's note: Springer Nature remains neutral with regard to jurisdictional claims in published maps and institutional affiliations.

\section{(c) (i)}

Open Access This article is licensed under a Creative Commons Attribution 4.0 International License, which permits use, sharing, adaptation, distribution and reproduction in any medium or format, as long as you give appropriate credit to the original author(s) and the source, provide a link to the Creative Commons license, and indicate if changes were made. The images or other third party material in this article are included in the article's Creative Commons license, unless indicated otherwise in a credit line to the material. If material is not included in the article's Creative Commons license and your intended use is not permitted by statutory regulation or exceeds the permitted use, you will need to obtain permission directly from the copyright holder. To view a copy of this license, visit http://creativecommons.org/ licenses/by/4.0/.

(c) The Author(s) 2018 